\title{
Clifford-Finsler Algebroids and Nonholonomic Einstein-Dirac Structures
}

\author{
Sergiu I. Vacaru* \\ Department of Mathematics, Brock University, \\ St. Catharines, Ontario, Canada L2S 3A1
}

March 5, 2006

\begin{abstract}
We propose a new framework for constructing geometric and physical models on nonholonomic manifolds provided both with Clifford Lie algebroid symmetry and nonlinear connection structure. Explicit parametrizations of generic off-diagonal metrics and linear and nonlinear connections define different types of Finsler, Lagrange and/or Riemann-Cartan spaces. A generalization to spinor fields and Dirac operators on nonholonomic manifolds motivates the theory of Clifford algebroids defined as Clifford bundles, in general, enabled with nonintegrable distributions defining the nonlinear connection. In this work, we elaborate the algebroid spinor differential geometry and formulate the (scalar, Proca, graviton, spinor and gauge) field equations on Lie algebroids. The paper communicates new developments in geometrical formulation of physical theories and this approach is grounded on a number of previous examples when exact solutions with generic offdiagonal metrics and generalized symmetries in modern gravity define nonholonomic spacetime manifolds with uncompactified extra dimensions.
\end{abstract}

Keywords: Lie algebroids, Clifford algebroids, Finsler and Lagrange geometry, exact solutions, Einstein-Dirac equations, string and Einstein gravity.

2000 AMS Subject Classification:

15A66, 17B99, 53A40, 58B20, 81R25, 83C20, 83C60, 83E99

\section{Introduction}

A class of spacetimes possessing noncommutative and/or Lie algebroid symmetries can be defined as exact solutions in string and Einstein gravity

*svacaru@brocku.ca 
[1, 2]. This leads to new developments in formulation of classical and quantum field theories following the geometry of nonholonomic manifolds 3] possessing Lie algebroid symmetry [4]. Lie algebroid structures in gravity are modelled by generic off-diagonal metrics and nonholonomic frames (vielbeins) with associated nontrivial nonlinear connection (N-connection). The spacetimes provided with compatible metric, linear connection and $\mathrm{N}$-connection structures and possessing Lie algebroid symmetry are called Einstein-Cartan algebroids, or (in a more general context, for various extensions of the Riemann-Cartan geometry) Lie N-algebroids. Usually, the Lie algebroids can be defined for a vector, or tangent, bundle but, in general, they can be considered for any nonholonomic manifold provided with a nonintegrable (nonholonomic) distribution ${ }^{1}$. In brief, such spaces are called Lie N-algebroids. Similar constructions elaborated for the Einstein-Dirac spaces give rise to the geometry of Clifford algebroids. If the curved spinor spaces are also enabled with Finsler, or Lagrange, structures, we deal with Clifford-Finsler, or Clifford-Lagrange, algebroids.

We note that the methods of Finsler and Lagrange geometry [5, 6] were recently reconsidered in a new way in order to solve physical problems related to standard theories of gravity and field interactions [1, 7, 6, 8, 9]. If the former physical applications of Finsler geometry were elaborated on tangent/ vector bundles, with less straightforward connections to standard physical theories (see reviews and references in [1, 7, 6]), in our approach we tried to define Finsler like structures as exact solutions in Einstein and extra dimension gravity [8, 9] when certain dimensions are not compactified. Such constructions are related to the geometry of nonholonomic manifolds possessing generalized symmetries (Lie algebroid and/or Clifford symmetries, noncommutative structures induces by anholonomic frames, ...) and a number of ideas and methods from Finsler geometry seem to be of general interest and significant importance for physical applications. Here we note, that this paper in not just on Clifford-Finsler geometry and related Lie algebroid structures but rather on (pseudo) Riemann geometry and gravitational and field interactions (and extensions to non-trivial torsion induced, for instance, from string theory and/or by nonholonomic frame effects) when the spinor and Lie aglebroid structures are defined in nonholonomic form and certain methods from Finsler geometry became very important and efficient in order to solve nonlinear physical problems.

This work develops the geometry of Clifford N-algebroids and generalized Finsler-spinor spaces elaborated in Refs. 11, 12, 13, 7]. If the first ap-

\footnotetext{
${ }^{1}$ In our works we use distributions defining $\mathrm{N}$-connection structures with the coefficients induced by the metric's off-diagonal terms and corresponding vielbein's coefficients. The geometric constructions are performed for nonholonomic manifolds, i. e. spaces provided with nonintegrable distributions. In a particular case, when such distributions are related to the exact sequences of subspaces defining a $\mathrm{N}$-connection, the spaces are called $\mathrm{N}$-anholonomic.
} 
plications of algebroid methods were in geometric mechanics [14, 15, 16, 17, the recent works suggest a very promising route toward the theory of gauge fields, gravity and strings and noncommutative geometry [7, 18, 1]. We cite Ref. 44 for details on algebroid theory and related bibliography.

In the present paper, we address essentially the following two purposes: The first one is to define and study the geometry of Clifford algebroids and their $\mathrm{N}$-anholonomic deformations, Clifford $\mathrm{N}$-algebroids, and analysis of theirs main properties in relation to spinors in gravity theories and on nonholonomic manifolds. The second aim is the formulation of the field equations on Lie algebroids.

The structure of the paper is the following: The theory of Clifford algebroids is formulated in section 2: we remember the main definitions of nonholonomic manifolds provided with $\mathrm{N}$-connection structure, define Clifford $\mathrm{N}$-algebroids and study the related spinor differential geometry. Section 3 is devoted to the field equations on $\mathrm{N}$-anholonomic manifolds and their redefinition on Clifford N-anholonomic algebroids. We start with a study of the Dirac operator and spin connections on nonholonomic manifolds. Then the constructions are completed with spinor formulations of the basic equations for scalar, Proca, graviton, Dirac and gauge fields interactions and related Lie/Clifford N-algebroid structures. In section 4, we present conclusions and outlook.

\section{Clifford Algebroids and N-Connections}

The geometry of spinor spaces enabled with nonlinear connection (N-connection) structure was elaborated in a series of works [11, 12, 13] (see also papers [19, 20, 21] for general references on Clifford and spinor differential geometry and applications to physics). Here we note that the concept of $\mathrm{N}$-connection was originally proposed in the framework of Finsler geometry and geometric mechanics but such nonholonomic structures ${ }^{2}$ may be also considered on (pseudo) Riemannian and Einstein-Cartan-Weyl spaces, see discussion and historical remarks in Ref. [7. A class of nonholonomic spinor configurations can be defined by exact solutions of the Einstein-Dirac equations parametrized by generic off-diagonal metric ansatz, nonholonomic vielbeins associated to nontrivial $\mathrm{N}$-connections and arbitrary linear connections with nontrivial torsion.

The aim of this section is to formulate the theory of Clifford algebroids provided with nonlinear connection ( $\mathrm{N}$-connection) structures, i. e. the theory of Clifford N-algebroids. For holonomic configurations, the Clifford algebroids can be defined as usual Lie algebroids [4 but associated to a Clifford bundle instead to a vector or tangent bundle.

\footnotetext{
${ }^{2}$ defined by exact sequences of subspaces of the tangent space to the spacetime manifold and related nonintegrable distributions
} 


\subsection{Nonholonomic manifolds and nonlinear conections}

We outline some basic definitions and formulas from the geometry of manifolds provided with $\mathrm{N}$-connection structure, see details in Refs. 6, 7].

Let us consider a Riemann-Cartan manifold $V$ of dimension $n+m$ and necessary smooth class and provided with general metric (of arbitrary signature) and linear connection structures. The local coordinates are denoted $u=(x, y)$, or $u^{\alpha}=\left(x^{i}, y^{a}\right)$, where the abstract, or coordinate, indices take respectively the values $i, j, k, \ldots=1,2, \ldots, n$ and $a, b, c, \ldots=$ $n+1, n+2, \ldots, n+m$. Such a splitting of dimension and coordinates will be adapted bellow to the nonlinear connection structure. We denote by $M$ a subspace of $V, \operatorname{dim} M=n$, provided with local coordinates $x^{i}$. The metric on $V$ is parametrized in the form

$$
\mathbf{g}=g_{\alpha \beta} \mathbf{e}^{\alpha} \otimes \mathbf{e}^{\beta}=g_{i j}(u) \mathbf{e}^{i} \otimes \mathbf{e}^{j}+h_{a b}(u) \mathbf{e}^{a} \otimes \mathbf{e}^{b}
$$

where

$$
\mathbf{e}^{\mu}=\left[e^{i}=d x^{i}, \mathbf{e}^{a}=d y^{a}+N_{i}^{a}(u) d x^{i}\right]
$$

is the dual frame to

$$
\mathbf{e}_{\nu}=\left[\mathbf{e}_{i}=\frac{\partial}{\partial x^{i}}-N_{i}^{a}(u) \frac{\partial}{\partial y^{a}}, e_{a}=\frac{\partial}{\partial y^{a}}\right] .
$$

Such vielbeins are called $\mathrm{N}$-adapted frames. ${ }^{3}$

We denote by $\pi^{\top}: T V \rightarrow T M$ the differential of a map $\pi: T V \rightarrow h V$, where $h V$ is locally isomorphic to $M$, defined by fiber preserving morphisms of the tangent bundles $T V$ and $T M$. The kernel of $\pi^{\top}$ is just the vertical subspace $v V, \operatorname{dim}(v V)=m$, with a related inclusion mapping $i: v V \rightarrow$ $T V$ and $h V$ is a horizontal subspace. It should be emphasized that one exists such maps and local decompositions when $V \rightarrow M$ is a surjective submersion. A particular case is that of a fiber bundle but we can obtain the results in the general case. ${ }^{4}$ A nonlinear connection ( $\mathrm{N}-$ connection) $\mathbf{N}$ on a manifold $V$ is defined by the splitting on the left of an exact sequence

$$
0 \rightarrow v V \rightarrow T V \rightarrow T V / v V \rightarrow 0
$$

i. e. by a morphism of submanifolds $\mathbf{N}: T V \rightarrow v V$ such that $\mathbf{N} \circ \mathbf{i}$ is the unity in $v V$.

\footnotetext{
${ }^{3}$ In order to preserve a relation with the previous denotations [7] 11] 12] 13, we note that $\mathbf{e}_{\nu}=\left(e_{i}, e_{a}\right)$ and $\mathbf{e}^{\mu}=\left(e^{i}, e^{a}\right)$ are, respectively, the former $\delta_{\nu}=\delta / \partial u^{\nu}=\left(\delta_{i}, \partial_{a}\right)$ and $\delta^{\mu}=\delta u^{\mu}=\left(d^{i}, \delta^{a}\right)$ which emphasize that operators (3) and (2) define, correspondingly, certain "N-elongated" partial derivatives and differentials which are more convenient for calculations on such nonholonomic manifolds.

${ }^{4}$ see discussions and references in 3] 7] related to almost sympletic manifolds, (pseudo) Riemannian spaces and vector bundles and generalizations
} 
Equivalently, a $\mathrm{N}$-connection is defined by a Whitney sum of horizontal (h) subspace, $h V \simeq M$ (we shall use the symbol " $\simeq$ " in order to emphasize some isomorphisms of spaces) and vertical (v) subspace, $v V$,

$$
T \mathbf{V}=h V \oplus v V .
$$

The spaces provided with $\mathrm{N}$-connection structure are denoted by boldface symbols. For instance, we write $\mathbf{V}$ for a manifold $V$ provided with a distribution (4) (being, in general, nonintegrable, i. e. nonholonomic ${ }^{5}$ ). Such manifolds are called $\mathrm{N}$-anholonomic with the nonholonomy defined by a $\mathrm{N}$-connection structure. In a similar manner, we can define nonholonomic manifolds enabled with certain more general nonintegrable (nonholonomic) distributions of subspaces in $T V$, or in $T T V$, and so on ... but in this paper we shall restrict our considerations only to $\mathrm{N}$-anholonomic manifolds with $\mathrm{N}$-connection splitting on $T V$. We shall use boldfaced indices for the geometric objects adapted to the $\mathrm{N}$-connection.

Locally, a $\mathrm{N}$-connection is defined by its coefficients $N_{i}^{a}(u)$,

$$
\mathbf{N}=N_{i}^{a}(u) d x^{i} \otimes \frac{\partial}{\partial y^{a}}
$$

The well known class of linear connections consists on a particular subclass with the coefficients being linear on $y^{a}$, i.e., $N_{i}^{a}(u)=\Gamma_{b j}^{a}(x) y^{b}$. Any Nconnection is characterized by its $\mathrm{N}$-connection curvature

$$
\boldsymbol{\Omega}=\frac{1}{2} \Omega_{i j}^{a} d x^{i} \wedge d x^{j} \otimes \frac{\partial}{\partial y^{a}}
$$

with N-connection curvature coefficients

$$
\Omega_{i j}^{a}=\delta_{[j} N_{i]}^{a}=\delta_{j} N_{i}^{a}-\delta_{i} N_{j}^{a}=\frac{\partial N_{i}^{a}}{\partial x^{j}}-\frac{\partial N_{j}^{a}}{\partial x^{i}}+N_{i}^{b} \frac{\partial N_{j}^{a}}{\partial y^{b}}-N_{j}^{b} \frac{\partial N_{i}^{a}}{\partial y^{b}},
$$

and states the condition that the vielbeins (2) satisfy the nonholonomy (equivalently, anholonomy) relations

$$
\left[\mathbf{e}_{\alpha}, \mathbf{e}_{\beta}\right]=\mathbf{e}_{\alpha} \mathbf{e}_{\beta}-\mathbf{e}_{\beta} \mathbf{e}_{\alpha}=W_{\alpha \beta}^{\gamma} \mathbf{e}_{\gamma}
$$

with (antisymmetric) nontrivial anholonomy coefficients $W_{i a}^{b}=\partial_{a} N_{i}^{b}$ and $W_{j i}^{a}=\Omega_{i j}^{a}$.

All our further geometric constructions will be for spaces with nonholonomic splitting (4) and performed in 'N-adapted' form with respect to local frames of type (2) and (3).

\footnotetext{
${ }^{5}$ in literature it is also used an equivalent term: anholonomic
} 


\subsection{Clifford $\mathrm{N}$-algebroids}

Let us state the notations for abstract (coordinate) d-tensor indices of geometrical objects defined with respect to an arbitrary (coordinate) local basis, i. e. system of reference. For a local basis on $\mathbf{V}$, we write $e_{\alpha}=\left(e_{i}, v_{a}\right)$. The small Greek indices $\alpha, \beta, \gamma, \ldots$ are considered to be general ones, running values $1,2, \ldots, n+m$ and $i, j, k, \ldots$ and $a, b, c, \ldots$ respectively label the geometrical objects on the base and typical "fiber" and run, correspondingly, the values $1,2, \ldots, n$ and $1,2, \ldots, m$. The dual base is denoted by $e^{\alpha}=\left(e^{i}, v^{a}\right)$. The local coordinates of a point $u \in \mathbf{V}$ are written $\mathbf{u}=(x, y)$, or $u^{\alpha}=\left(x^{i}, y^{a}\right)$, where $y^{a}$ is the $a$-th coordinate with respect to the basis $\left(v_{a}\right)$ and $\left(x^{i}\right)$ are local coordinates on $h \mathbf{V}$ with respect to $e_{i}$. We shall use "boldface" symbols in order to emphasize that the objects are defined on spaces provided with $\mathrm{N}$-connection structure.

We suppose that the $\mathrm{N}$-anholonomic manifold $\mathbf{V}$ admits a $\mathrm{d}$-spinor structure which allows us to introduce spinor coordinates and parametrizations of geometrical objects. Let

$$
\mathbf{e}_{\dot{\alpha}}{ }^{\dot{\alpha}}=\left(e_{i}{ }^{\dot{\mathbf{1}}}, e_{i}{ }^{\dot{2}}, \ldots, e_{i} \stackrel{\tilde{\mathbf{k}}}{(n)}, e_{a}{ }^{\dot{\mathbf{1}}}, e_{\dot{a}}{ }^{\dot{2}}, \ldots, e_{\dot{a}} \tilde{\mathbf{\mathbf { k }}}_{(m)}\right)
$$

with boldfaced indices running coordinate values on dimensions of $\mathrm{d}$-spinor spaces, $\tilde{\mathbf{k}}_{(n)}$ and $\tilde{\mathbf{k}}_{(m)}$, be the coefficients of a d-spinor basis

$$
\mathbf{e}_{\dot{\alpha}}=\left(e_{i}, e_{a}\right) \text {. }
$$

The dual basis (co-basis)

$$
\mathbf{e}^{\dot{\alpha}}=\left(e^{i}, e^{\dot{a}}\right)
$$

has the coefficients

$$
\mathbf{e}_{\dot{\alpha}}{ }^{\alpha}=\left(e_{\mathbf{i}}{ }^{i}, e_{\dot{\mathbf{2}}}{ }^{i}, \ldots, e_{\tilde{\mathbf{k}}_{(n)}}^{i}, e_{\mathbf{i}}{ }^{a}, e_{\dot{\mathbf{2}}}{ }^{a}, \ldots, e_{\tilde{\mathbf{k}}_{(m)}}{ }^{a}\right) .
$$

Similar formulas hold for the associated $\mathrm{d}$-spinor spaces provided with local bases $\mathbf{e}_{\grave{\alpha}}{ }^{\grave{\alpha}}$ and $\mathbf{e}_{\grave{\alpha}}{ }^{\grave{\alpha}}$. Such spinor bases are stated to be compatible to the Nconnection splitting, i. e. to the vielbeins (3) and (2). For a given $\mathrm{d}-$-metric structure on $\mathbf{V}$ and its spinor decomposition, with associated spinor bases $\mathbf{e}_{\grave{\alpha}}=\left(e_{\grave{\imath}}, e_{\grave{a}}\right)$, which allows us to introduce the $\gamma$-objects, we can define, for instance, a $\mathrm{N}$-adapted basis

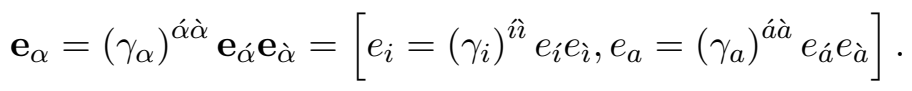

As a result, we can alternatively consider spinor coordinates, for instance,

$$
u^{\alpha}=\left(x^{i}, y^{a}\right) \rightarrow u^{\grave{\alpha} \grave{\alpha}}=\left(x^{\grave{\imath}}, y^{a ́ a ̀}\right) .
$$

For even dimensions of $n$, or $m$, further reductions are possible, when $x^{\imath \grave{\imath}} \rightarrow$ $x^{I I^{\prime}}$, or $y^{a \grave{a}} \rightarrow y^{A A^{\prime}}$. This way, the d-tensor indices can be transformed into the d-spinor ones and inversely. 
The standard definition of a Lie algebroid $\mathcal{A} \doteqdot(E,[\cdot, \cdot], \rho)$ is associated a vector bundle $\mathcal{E}=(E, \pi, M)$, with a surjective map $\pi: E \longrightarrow M$ of the total spaces $E$ to the base manifold $M$, of respective dimensions $\operatorname{dim} E=$ $n+m$ and $\operatorname{dim} M=n$. The algebroid structure is stated by the anchor map $\rho: E \rightarrow T M(T M$ is the tangent bundle to $M)$ and a Lie bracket on the $C^{\infty}(M)$-module of sections of $E$, denoted $\operatorname{Sec}(E)$, such that

$$
[X, f Y]=f[X, Y]+\rho(X)(f) Y
$$

for any $X, Y \in \operatorname{Sec}(E)$ and $f \in C^{\infty}(M)$. The anchor also induces a homomorphism of $C^{\infty}(M)$-modules $\rho: \operatorname{Sec}(A) \rightarrow \mathcal{X}^{1}(M)$ where $\wedge^{r}(M)$ and $\mathcal{X}^{r}(M)$ will denote, respectively, the spaces of differential $r$-forms and $r$ multivector fields on $M$.

In local form, the Lie algebroid structure on the manifold $\mathbf{V}$ is defined by its structure functions $\rho_{a}^{i}(x)$ and $C_{a b}^{f}(x)$ defining the relations

$$
\begin{aligned}
\rho\left(e_{a}\right) & =\rho_{a}^{i}(x) e_{i}=\rho_{a}^{i}(x) \partial_{\underline{i}}, \\
{\left[e_{a}, e_{b}\right] } & =C_{a b}^{c}(x) e_{c}
\end{aligned}
$$

and subjected to the structure equations

$$
\rho_{a}^{j} \frac{\partial \rho_{b}^{i}}{\partial x^{j}}-\rho_{b}^{j} \frac{\partial \rho_{a}^{i}}{\partial x^{j}}=\rho_{c}^{j} C_{a b}^{c} \text { and } \sum_{c y c l i c(a, b, c)}\left(\rho_{a}^{j} \frac{\partial C_{b c}^{d}}{\partial x^{j}}+C_{a f}^{d} C_{b c}^{f}\right)=0
$$

for simplicity, we shall omit underlying of coordinate indices if it will not result in ambiguities. Such equations are standard ones for the Lie algebroids but defined on a $\mathrm{N}$-anholonomic manifolds. In brief, we call them Lie Nalgebroids.

Definition 2.1 A Clifford algebroid $\mathcal{C}(E) \doteqdot\left(\mathbb{C l}(E),{ }^{s}[\cdot, \cdot],{ }^{s} \rho\right)$ is associated to a Clifford bundle $\mathbb{C l}(E) \doteq \mathbb{C l}\left(T^{*} E\right)$ defined by the vector bundle $\mathcal{E}=(E, \pi, M)$ and provided with "spin" anchor $s$ and (Lie type) commutator structure ${ }^{s}[\cdot, \cdot]$ defined on the Clifford module $\operatorname{Sec}(\mathbb{C l}(M))$.

The Clifford algebroid strucure on a manifold $M$ is defined $\mathcal{C}(T M) \doteqdot$ $\left(\mathbb{C l}(T M),{ }^{s}[\cdot, \cdot],{ }^{s} \rho\right)$.

In local form, the spinor structure functions are written

$$
\begin{aligned}
& \rho\left(e_{a \dot{a}}\right)=\rho_{a \grave{a}}^{i}(x) e_{i}=\rho_{\grave{a} \grave{a}}^{\underline{i}}(x) \partial_{\underline{i}},
\end{aligned}
$$

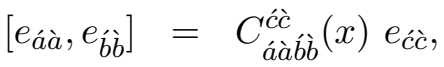

where we can consider a spinor decomposition on $M$ with re-definition of indices like $i \rightarrow \imath$, $\imath$. Such structure functions can be induced by pure spinor ones,

$$
\rho\left(e_{a}\right)=\rho_{a}^{i}(x) e_{i}
$$


and

$$
\left[e_{a}, e_{\hat{b}}\right]=C_{\dot{a} \dot{b}}^{\dot{c}}(x) e_{\dot{c}},
$$

where, for instance, we can consider $\rho_{a \grave{a}}^{i}(x)=\rho_{\grave{a}}^{i}(x) \rho_{\grave{a}}^{i}(x)$ for any fixed value of $i$. The structure equations (9) can be written in spinor form by introducing spinor variables (see examples of calculus with spinors in the next section).

We can write down the Lie algebroid and $\mathrm{N}$-connection structures in a compatible form by introducing the "N-adapted" anchor

$$
\widehat{\rho}_{a}^{j}(x, u) \doteqdot \mathbf{e}_{\underline{j}}^{j}(x, u) \mathbf{e}_{a}^{a}(x, u) \rho_{\underline{a}}^{\underline{j}}(x)
$$

and "N-adapted" (boldfaced) structure functions

$$
\mathbf{C}_{a g}^{f}(x, u)=\mathbf{e}_{\underline{f}}^{f}(x, u) \mathbf{e}_{a}^{\underline{a}}(x, u) \mathbf{e}_{g}^{\underline{g}}(x, u) C_{\underline{a} \underline{g}}^{\underline{f}}(x),
$$

respectively, into formulas (7), (8) and (9). In general, the RC-algebroids are defined by the corresponding sets of functions $\hat{\rho}_{a}^{j}(x, y)$ and $\mathbf{C}_{a g}^{f}(x, y)$ with additional dependencies on $\mathrm{v}$-variables $y^{b}$ for the $\mathrm{N}$-adapted structure functions. For such Lie N-algebroids, the structure relations became

$$
\begin{aligned}
\widehat{\rho}\left(e_{b}\right) & =\widehat{\rho}_{b}^{i}(x, y) e_{i}, \\
{\left[e_{d}, e_{b}\right] } & =\mathbf{C}_{d b}^{f}(x, y) e_{f}
\end{aligned}
$$

and the structure equations of the Lie $\mathrm{N}$-algebroid are written

$$
\begin{aligned}
\hat{\rho}_{a}^{j} e_{j}\left(\widehat{\rho}_{b}^{i}\right)-\widehat{\rho}_{b}^{j} e_{j}\left(\widehat{\rho}_{a}^{i}\right) & =\hat{\rho}_{e}^{j} \mathbf{C}_{a b}^{e}, \\
\sum_{\text {cyclic }(a, b, e)}\left(\hat{\rho}_{a}^{j} e_{j}\left(\mathbf{C}_{b e}^{f}\right)+\mathbf{C}_{a g}^{f} \mathbf{C}_{b e}^{g}-\mathbf{C}_{b^{\prime} e^{\prime}}^{f^{\prime}} \widehat{\rho}_{a}^{j} \mathbf{Q}_{f^{\prime} b e j}^{f b^{\prime} e^{\prime}}\right) & =0
\end{aligned}
$$

for $\mathbf{Q}_{f^{\prime} b e j}^{f b^{\prime} e^{\prime}}=\mathbf{e}_{\underline{b}}^{b^{\prime}} \mathbf{e}_{\underline{e}}^{e^{\prime}} \mathbf{e}_{f^{\prime}} e_{j}\left(\mathbf{e}_{b} \underline{b} \mathbf{e}_{e}{ }^{\underline{e}} \mathbf{e}_{\underline{f}}^{f}\right)$ with the values $\mathbf{e}_{\underline{b}}^{b^{\prime}}$ and $\mathbf{e}_{f^{\prime}}$ defined by the $\mathrm{N}$-connection. The Lie $\mathrm{N}$-algebroid structure will be characterized by the data $\widehat{\rho}_{b}^{i}(x, y)$ and $\mathbf{C}_{d b}^{f}(x, y)$ stated with respect to the $\mathrm{N}$-adapted frames (3) and (2).

A Riemann-Cartan algebroid (in brief, RC-algebroid) is a Lie algebroid $\mathcal{A} \doteqdot(\mathbf{V},[\cdot, \cdot], \rho)$ associated to a $\mathrm{N}$-anholonomic manifold $\mathbf{V}$ provided with a $\mathrm{N}$-connection $\mathbf{N}$, symmetric metric $\mathbf{g}(\mathbf{u})$ and linear connection $\boldsymbol{\Gamma}(\mathbf{u})$ structures resulting in a metric compatible and $\mathrm{N}$-adapted covariant derivative $\mathbf{D}$, when $\mathbf{D g}=\mathbf{0}$, but, in general, with nonvanishing torsion. In spinor variables, the $\mathrm{RC}$-algebroids transform into Clifford $\mathrm{N}$-algebroids associated to corresponding $\mathrm{N}$-anholonomic manifolds instead of vector bundles. They are characterized by the same set of relations (13)-(17) re-written in d-spinor variables. 


\section{$2.3 \quad \mathrm{~N}$-algebroid spinor differential geometry}

The goal of the section is to outline the main results from the differential geometry of $\mathrm{d}$-spinors for the Clifford $\mathrm{N}$-algebroids and related $\mathrm{N}-$ anholonomic manifolds. The $\mathrm{d}$-tensor and $\mathrm{d}$-connection formulas and basic equations are investigated in details in Ref. [2]. Such Lie N-algebroid relations can be obtained by "anchoring" the formulas for $\mathrm{d}$-connections, $\mathrm{d}$-torsions and $\mathrm{d}$-curvatures stated. In result, one obtains certain differential geometric objects on the set of sections like $\operatorname{Sec}(v \mathbf{V})$ or $\operatorname{Sec}(\mathbf{E})$, when the "fiber" derivatives are changed into horizontal ones, $\partial / \partial y^{a} \rightarrow \rho_{a}^{i} \partial / \partial x^{a}$, or in N-adapted form, $e_{a} \rightarrow \widehat{\rho}_{a}^{j} e_{j}$. In spinor/d-spinor variables, such formulas transform into certain analogous on Clifford $\mathrm{N}$-algebroids provided with arbitrary but $\mathrm{N}$-adapted and compatible $\mathrm{d}$-metric and $\mathrm{d}$ - connection structure.

We use denotations

$$
e^{\alpha}=\left(e^{i}, e^{a}\right) \in \gamma^{\alpha}=\left(\gamma^{i}, \gamma^{a}\right) \text { and } \zeta^{\dot{\alpha}}=\left(\zeta^{i}, \zeta^{\dot{a}}\right) \in \gamma^{\dot{\alpha}}=\left(\gamma^{i}, \gamma^{\dot{a}}\right)
$$

for, respectively, elements of modules of d-vector and irreduced d-spinor fields (see details in [11]). D-tensors and $\mathrm{d}$-spinor tensors (irreduced or reduced) will be interpreted as elements of corresponding $\gamma$-modules, for instance,

$$
q_{\beta \ldots}^{\alpha} \in \gamma_{\beta}^{\alpha}, \psi_{\beta}^{\dot{\alpha}} \quad \stackrel{\gamma}{\ldots}^{\alpha} \in \gamma_{\dot{\beta}}^{\alpha}{ }_{\ldots}^{\dot{\alpha}}, \xi_{J K^{\prime} N^{\prime}}^{I I^{\prime}} \in \gamma_{J K^{\prime} N^{\prime}}^{I I^{\prime}}, \ldots
$$

We can establish a correspondence between the $\mathrm{d}$-metric $g_{\alpha \beta}$ (11) and d-spinor metric $\epsilon_{\dot{\alpha} \dot{\beta}}$ for both h- and v-subspaces of $\mathbf{V}$ ) by using the relation

$$
g_{\alpha \beta}=\frac{1}{\tilde{k}_{(n)}+\tilde{k}_{(m)}}\left(\left(\gamma_{(\alpha}(u)\right)^{\alpha \grave{\alpha}}\left(\gamma_{\beta)}(u)\right)^{\dot{\beta} \grave{\beta}}\right) \epsilon_{\dot{\alpha} \grave{\beta}} \epsilon_{\dot{\beta} \grave{\alpha}}
$$

where $(\alpha \beta)$ denotes symmetrization on such indices and

$$
\left(\gamma_{\alpha}(u)\right)^{\alpha \dot{\alpha}}=l_{\alpha}^{\widehat{\alpha}}(u)\left(\gamma_{\widehat{\alpha}}\right)^{\alpha ́ \grave{\alpha}} .
$$

In brief, we can write (18) in the form

$$
g_{\alpha \beta}=\epsilon_{\dot{\alpha} \grave{\beta}} \epsilon_{\dot{\beta} \dot{\alpha}}
$$

if the $\gamma$-objects are considered as a fixed structure, whereas $\epsilon$-objects are treated as caring the metric "dynamics". This variant is used, for instance, in the so-called 2-spinor geometry [19, 20] and should be preferred if we have to make explicit the algebraic symmetry properties of d-spinor objects. An alternative way is to consider as fixed the algebraic structure of $\epsilon$-objects and to use variable components of $\gamma$-objects of type (19) for developing a variational d-spinor approach to gravitational and matter field interactions (the spinor Ashtekar variables [26] are introduced in this manner). In this 
paper we shall follow in the bulk the first approach but we note that the second type of spinor calculus is more convenient for finding exact solutions with nonholonomic variables.

We note that a d-spinor metric

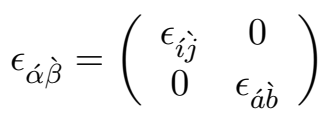

on the d-spinor space $\mathbf{S}=\left(S_{(h)}, S_{(v)}\right)$ may have symmetric or antisymmetric h $(\mathrm{v})$-components $\epsilon_{\hat{i} j}\left(\epsilon_{\dot{a} \grave{b}}\right)$. For simplicity, in this section (in order to avoid cumbersome calculations connected with eight-fold periodicity on dimensions $n$ and $m$ on a $\mathrm{N}$-anholonomic manifold) we shall develop a general d-spinor formalism only by using irreduced spinor spaces $\mathbf{S}_{(h)}$ and $\mathbf{S}_{(h)}^{\prime}$.

\subsubsection{D-covariant derivation}

For a d-covariant operator

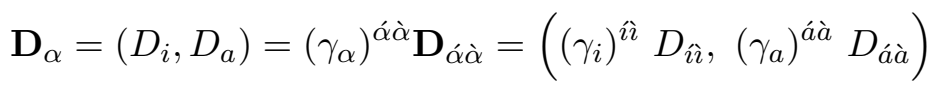

(in brief, we shall write

$$
\left.\mathbf{D}_{\alpha}=\mathbf{D}_{\dot{\alpha} \grave{\alpha}}=\left(D_{\grave{\imath} \grave{i}}, D_{\dot{a} \grave{a}}\right)\right)
$$

being constructed by using the coefficients of a d-connection, we define the action on a d-spinor $\gamma^{\dot{\beta}}$ as a map

$$
\mathbf{D}_{\dot{\alpha} \grave{\alpha}}: \gamma^{\dot{\beta}} \rightarrow \gamma_{\alpha}^{\dot{\beta}}=\gamma_{\dot{\alpha} \grave{\alpha}}^{\dot{\beta}}
$$

satisfying conditions

$$
\mathbf{D}_{\alpha}\left(\xi^{\dot{\beta}}+\eta^{\dot{\beta}}\right)=\mathbf{D}_{\alpha} \xi^{\dot{\beta}}+\mathbf{D}_{\alpha} \eta^{\dot{\beta}} \text { and } \mathbf{D}_{\alpha}\left(f \xi^{\dot{\beta}}\right)=f \mathbf{D}_{\alpha} \xi^{\dot{\beta}}+\xi^{\dot{\beta}} \mathbf{D}_{\alpha} f,
$$

for every $\xi^{\dot{\beta}}, \eta^{\dot{\beta}} \in \gamma^{\dot{\beta}}$ and $f$ being a scalar field on $\mathbf{V}$. It is also required that one holds the Leibnitz rule

$$
\left(\mathbf{D}_{\alpha} \zeta_{\dot{\beta}}\right) \eta^{\dot{\beta}}=\mathbf{D}_{\alpha}\left(\zeta_{\dot{\beta}} \eta^{\dot{\beta}}\right)-\zeta_{\dot{\beta}} \mathbf{D}_{\alpha} \eta^{\dot{\beta}}
$$

and that $\mathbf{D}_{\alpha}$ is a real operator, i. e. it commuters with the operation of complex conjugation:

$$
\overline{\mathbf{D}_{\alpha} \psi_{\underline{\alpha} \underline{\beta \gamma} \ldots}}=\mathbf{D}_{\alpha}\left(\bar{\psi}_{\underline{\alpha} \underline{\beta \gamma} \ldots}\right) .
$$

Let us now analyze the question on uniqueness of action on $\mathrm{d}$-spinors of an operator $\mathbf{D}_{\alpha}$ satisfying some necessary conditions. Denoting by $\mathbf{D}_{\alpha}^{(1)}$ and $\mathbf{D}_{\alpha}$ two such d-covariant operators, we consider the map

$$
\left(\mathbf{D}_{\alpha}^{(1)}-\mathbf{D}_{\alpha}\right): \gamma^{\dot{\beta}} \rightarrow \gamma_{\dot{\alpha} \grave{\alpha}}^{\dot{\beta}} .
$$


Because the action on a scalar $f$ of both operators $\mathbf{D}_{\alpha}^{(1)}$ and $\mathbf{D}_{\alpha}$ must be identical, i.e.

$$
\mathbf{D}_{\alpha}^{(1)} f=\mathbf{D}_{\alpha} f,
$$

the action (21) on $f=\omega_{\dot{\beta}} \xi^{\dot{\beta}}$ must be written as

$$
\left(\mathbf{D}_{\alpha}^{(1)}-\mathbf{D}_{\alpha}\right)\left(\omega_{\dot{\beta}} \xi^{\dot{\beta}}\right)=0 .
$$

We conclude that there is an element $\Theta_{\alpha \dot{\alpha} \dot{\alpha} \hat{\beta}} \in \gamma_{\dot{\alpha} \alpha \dot{\alpha}}^{\dot{\gamma}}$ for which

$$
\mathbf{D}_{\dot{\alpha} \dot{\alpha}}^{(1)} \xi^{\dot{\gamma}}=\mathbf{D}_{\dot{\alpha} \dot{\alpha}} \xi^{\dot{\gamma}}+\Theta_{\dot{\alpha} \grave{\alpha} \dot{\beta}} \dot{\gamma}^{\dot{\beta}}
$$

and

$$
\mathbf{D}_{\dot{\alpha} \dot{\alpha}}^{(1)} \omega_{\dot{\beta}}=\mathbf{D}_{\dot{\alpha} \dot{\alpha}} \omega_{\dot{\beta}}-\Theta_{\dot{\alpha} \grave{\alpha} \dot{\gamma}} \dot{\gamma} \omega_{\dot{\gamma}}
$$

The action of the operator (21) on a d-vector $v^{\beta}=v^{\dot{\beta} \grave{\beta}}$ can be written by using formula (23) for both indices $\dot{\beta}$ and $\grave{\beta}$ :

$$
\begin{aligned}
\left(\mathbf{D}_{\alpha}^{(1)}-\mathbf{D}_{\alpha}\right) v^{\dot{\beta} \grave{\beta}} & =\Theta_{\alpha \dot{\gamma}}{ }^{\dot{\beta}} v^{\dot{\gamma} \grave{\beta}}+\Theta_{\alpha \grave{\gamma}}{ }^{\grave{\beta}} v^{\dot{\beta} \grave{\gamma}} \\
& =\left(\Theta_{\alpha \dot{\gamma}} e_{\grave{\gamma}}^{\grave{\beta}}+\Theta_{\alpha \grave{\gamma}} e_{\grave{\gamma}}^{\grave{\beta}}\right) v^{\dot{\gamma} \dot{\gamma}}=Q_{\alpha \gamma}^{\beta} v^{\gamma},
\end{aligned}
$$

where

$$
Q_{\alpha \gamma}^{\beta}=Q_{\alpha \dot{\alpha} \grave{\alpha} \dot{\gamma}}^{\dot{\beta} \grave{\gamma}}=\Theta_{\alpha \dot{\gamma}}{ }^{\dot{\beta}} e_{\grave{\gamma}}^{\grave{\beta}}+\Theta_{\alpha \grave{\gamma}}^{\grave{\beta}} e_{\dot{\gamma}}^{\dot{\beta}} \text {. }
$$

The commutator $\mathbf{D}_{[\alpha} \mathbf{D}_{\beta]}$ defines the d-torsion. Applying operators $\mathbf{D}_{[\alpha}^{(1)} \mathbf{D}_{\beta]}^{(1)}$ and $\mathbf{D}_{[\alpha} \mathbf{D}_{\beta]}$ on $f=\omega_{\dot{\beta}} \xi^{\dot{\beta}}$, we can write

$$
T_{\alpha \beta}^{(1) \gamma}-T_{\alpha \beta}^{\gamma}=Q_{\beta \alpha}^{\gamma}-Q_{\alpha \beta}^{\gamma}
$$

with $Q_{\alpha \beta}^{\gamma}$ from (24).

The action of operator $\mathbf{D}_{\alpha}^{(1)}$ on d-spinor tensors must be constructed by using formula (23) for every upper indices and formula (24) for every lower indices.

\subsubsection{N-adapted Infeld - van der Waerden coefficients}

A d-spinor $\kappa^{\dot{\alpha}} \in \gamma^{\alpha}$ has the components $\kappa^{\dot{\alpha}}=\kappa^{\dot{\alpha}} \mathbf{e}_{\dot{\alpha}}^{\dot{\alpha}}=\left(\kappa^{i}, \kappa^{\dot{a}}\right)$ defined with respect to the $\mathrm{N}$-adapted spinor basis (5). Taking into account that

$$
\mathbf{e}_{\dot{\alpha}}^{\dot{\alpha}} \mathbf{e}_{\grave{\beta}}^{\grave{\beta}} \mathbf{D}_{\dot{\alpha} \grave{\beta}}=\mathbf{D}_{\dot{\alpha} \grave{\beta}},
$$

we compute the components $\mathbf{D}_{\dot{\alpha} \dot{\beta}} \kappa^{\underline{\gamma}}$,

$$
\begin{aligned}
\mathbf{e}_{\dot{\alpha}}^{\alpha} \mathbf{e}_{\grave{\beta}}^{\grave{\beta}} \mathbf{e}_{\dot{\gamma}}^{\dot{\gamma}} \mathbf{D}_{\dot{\alpha} \dot{\beta}} \kappa^{\dot{\gamma}} & =\mathbf{e}_{\hat{\epsilon}}^{\dot{\tau}} \mathbf{e}_{\dot{\gamma}}^{\dot{\gamma}} \mathbf{D}_{\dot{\alpha} \dot{\beta}} \kappa^{\dot{\epsilon}}+\kappa^{\dot{\epsilon}} \mathbf{e}_{\dot{\epsilon}}^{\dot{\gamma}} \mathbf{D}_{\dot{\alpha} \dot{\beta}} \mathbf{e}_{\dot{\epsilon}}^{\epsilon^{\epsilon}} \\
& =\mathbf{D}_{\dot{\alpha} \grave{\beta}} \kappa^{\dot{\gamma}}+\kappa^{\bar{\epsilon}} \varpi^{\dot{\gamma}} \dot{\beta} \dot{\epsilon}{ }^{\prime}
\end{aligned}
$$


where the coordinate components of the $\mathrm{d}$-spinor connection are defined

$$
\varpi_{\alpha \dot{\beta} \dot{\epsilon}}^{\dot{\gamma}} \doteq \mathbf{e}_{\dot{\tau}}^{\dot{\gamma}} \mathbf{D}_{\dot{\alpha} \dot{\beta}} \mathbf{e}_{\hat{\epsilon}}^{\dot{\tau}}
$$

We call the Infeld - van der Waerden d-symbols a set of objects $\varpi^{\dot{\gamma}}{ }_{\alpha} \dot{\beta} \dot{\epsilon}$ parametrized with respect to a coordinate d-spinor basis. Defining $\mathbf{D}_{\alpha}{ }^{\alpha} \hat{\beta} \hat{\epsilon}$ $\left(\gamma_{\alpha}\right)^{\dot{\alpha} \grave{\beta}} \mathbf{D}_{\alpha \grave{\alpha} \hat{\beta}}$, introducing denotations $\varpi_{\alpha \dot{\tau}}^{\dot{\gamma}} \doteq \varpi_{\dot{\alpha} \grave{\beta} \dot{\tau}}^{\dot{\gamma}}\left(\gamma_{\alpha}\right)^{\dot{\alpha} \grave{\beta}}$ and using properties (25), we write the relations

$$
l_{\alpha}^{\alpha} \mathbf{e}_{\dot{\beta}}^{\dot{\beta}} \mathbf{D}_{\alpha} \kappa^{\dot{\beta}}=\mathbf{D}_{\alpha} \kappa^{\dot{\beta}}+\kappa^{\dot{\delta}} \varpi_{\alpha \dot{\delta}}^{\dot{\beta}}
$$

and

$$
l_{\alpha}^{\alpha} \mathbf{e}_{\dot{\beta}}^{\dot{\beta}} \mathbf{D}_{\alpha} \mu_{\dot{\beta}}=\mathbf{D}_{\alpha} \mu_{\dot{\beta}}-\mu_{\dot{\delta}} \varpi_{\alpha \dot{\beta}}^{\dot{\delta}}
$$

for d-covariant derivations $\mathbf{D}_{\alpha} \kappa^{\dot{\beta}}$ and $\mathbf{D}_{\alpha} \mu_{\dot{\beta}}$.

We can consider expressions similar to (27) and (28) for values having both types of d-spinor and d-tensor indices, for instance,

$$
l_{\alpha}^{\alpha} l_{\gamma}^{\gamma} \mathbf{e}_{\dot{\delta}}^{\dot{\delta}} \mathbf{D}_{\alpha} \theta_{\dot{\delta}}^{\gamma}=\mathbf{D}_{\alpha} \theta_{\dot{\delta}}^{\gamma}-\theta_{\dot{\epsilon}}^{\gamma} \varpi_{\alpha \dot{\delta}}^{\dot{\epsilon}}+\theta_{\dot{\delta}}^{\tau} \Gamma^{\gamma}{ }_{\alpha \tau}
$$

(we can prove this by a straightforward calculation of the derivation $\mathbf{D}_{\alpha}\left(\theta_{\tilde{\delta}}{ }^{\tau}\right.$ $\left.\left.\mathbf{e}_{\dot{\delta}}^{\delta} l_{\tau}^{\gamma}\right)\right)$.

Now we shall consider some possible relations between components of $\mathrm{d}$-connections $\varpi_{\alpha \dot{\delta}}^{\dot{\epsilon}}$ and $\Gamma_{\alpha \tau}^{\gamma}$ and derivations of $\left(\gamma_{\alpha}\right)^{\dot{\alpha} \grave{\beta}}$. We can write

$$
\begin{aligned}
& \Gamma_{\beta \tau}^{\alpha}=l_{\alpha}^{\alpha} \mathbf{D}_{\tau} l_{\beta}^{\alpha}=l_{\alpha}^{\alpha} \mathbf{D}_{\tau}\left(\gamma_{\beta}\right)^{\epsilon \grave{\epsilon}}=l_{\alpha}^{\alpha} \mathbf{D}_{\tau}\left(\left(\gamma_{\beta}\right)^{\hat{\epsilon} \grave{\tau}} \mathbf{e}_{\hat{\epsilon}}^{\epsilon^{\epsilon}} \mathbf{e}_{\grave{\tau}}^{\bar{\tau}}\right) \\
& =l_{\alpha}^{\alpha} \mathbf{e}_{\dot{\alpha}}^{\alpha} \mathbf{e}_{\dot{\epsilon}}^{\grave{\epsilon}} \mathbf{D}_{\tau}\left(\gamma_{\beta}\right)^{\dot{\alpha} \grave{\epsilon}}+l_{\alpha}^{\alpha}\left(\gamma_{\beta}\right)^{\hat{\epsilon} \hat{\tau}}\left(\mathbf{e}_{\grave{\tau}}^{\grave{\tau}} \mathbf{D}_{\tau} \mathbf{e}_{\dot{\epsilon}}^{\dot{\epsilon}}+\mathbf{e}_{\hat{\epsilon}}^{\epsilon^{\epsilon}} \mathbf{D}_{\tau} \mathbf{e}_{\grave{\tau}}^{\grave{\tau}}\right) \\
& =l_{\dot{\alpha} \grave{\epsilon}}^{\alpha} \mathbf{D}_{\tau}\left(\gamma_{\beta}\right)^{\alpha \hat{\epsilon}}+l_{\dot{\alpha} \hat{\epsilon}}^{\alpha} \mathbf{e}_{\alpha}^{\dot{\alpha}} \mathbf{e}_{\grave{\epsilon}}^{\grave{\epsilon}}\left(\gamma_{\beta}\right)^{\hat{\epsilon} \grave{\tau}}\left(\mathbf{e}_{\grave{\tau}}^{\grave{\tau}} \mathbf{D}_{\tau} \mathbf{e}_{\dot{\epsilon}^{\epsilon}}^{\dot{\epsilon}}+\mathbf{e}_{\hat{\epsilon}}^{\epsilon^{\epsilon}} \mathbf{D}_{\tau} \mathbf{e}_{\grave{\tau}}^{\grave{\tau}}\right) \text {, }
\end{aligned}
$$

where $l_{\alpha}^{\alpha}=\left(\gamma_{\dot{\alpha} \alpha}\right)^{\alpha}$, from which one follows

$$
\left(\gamma_{\alpha}\right)^{\mu \hat{\nu}}\left(\gamma^{\beta}\right)_{\dot{\alpha} \hat{\beta}} \Gamma_{\gamma \beta}^{\alpha}=\left(\gamma^{\beta}\right)_{\dot{\alpha} \dot{\beta}} \mathbf{D}_{\gamma}\left(\gamma_{\beta}\right)^{\dot{\mu} \grave{\nu}}+\mathbf{e}_{\dot{\beta}}^{\grave{\nu}} \varpi_{\gamma \dot{\alpha}}^{\dot{\mu}}+\mathbf{e}_{\dot{\alpha}}^{\dot{\mu}} \varpi_{\gamma \grave{\beta}}^{\grave{\nu}} .
$$

Contracting the last expression on $\grave{\nu}$ and $\grave{\beta}$ and using an orthonormalized $\mathrm{d}$-spinor basis when $\varpi_{\gamma \dot{\beta}}^{\dot{\beta}}=0$ (a consequence from (26) ), we have

$$
\varpi_{\gamma \dot{\alpha}}^{\dot{\mu}}=\frac{1}{\tilde{k}_{(n)}+\tilde{k}_{(m)}}\left(\Gamma_{\gamma \dot{\alpha} \grave{\beta}}^{\dot{\mu} \grave{\beta}}-\left(\gamma^{\beta}\right)_{\dot{\alpha} \dot{\beta}} \mathbf{D}_{\gamma}\left(\gamma_{\beta}\right)^{\dot{\mu} \grave{\beta}}\right),
$$

where

$$
\Gamma_{\gamma \dot{\alpha} \grave{\beta}}^{\dot{\mu} \grave{\beta}}=\left(\gamma_{\alpha}\right)^{\dot{\mu} \grave{\beta}}\left(\gamma^{\beta}\right)_{\dot{\alpha} \grave{\beta}} \Gamma_{\gamma \beta}^{\alpha} .
$$

The d-spinor connection (30) can be defined by various type of of d-connections, inclusively, by the canonical one, see [1]. Such formulas can be applied 
on Clifford algebroid $\mathcal{C}(E) \doteqdot\left(\mathbb{C l}(E),{ }^{s}[\cdot, \cdot],{ }^{s} \rho\right)$ or on a Clifford $\mathrm{N}$-aglebroid $\mathcal{C}(v \mathbf{V}) \doteqdot\left(\mathbb{C l}(v \mathbf{V}),{ }^{s}[\cdot, \cdot],{ }^{s} \rho\right)$. We have to change the $\mathrm{v}$-derivatives into anchored ones, $\partial / \partial y^{a} \rightarrow \rho_{a}^{i} \partial / \partial x^{a}$, or in N-adapted form, $e_{a} \rightarrow \widehat{\rho}_{a}^{j} e_{j}$, and put the results in formulas (29) and (30). In result, one defines a canonical covariant spinor differential calculus, adapted to the $\mathrm{N}$-connection structure, acting on the set of sections $\operatorname{Sec}(\mathbf{E})$ or $\operatorname{Sec}(v \mathbf{V})$.

\subsubsection{D-spinors of curvature and torsion on $\mathrm{N}$-anholonomic manifolds}

The d-tensor indices of the commutator $\Delta_{\alpha \beta}$ can be transformed into dspinor ones:

$$
\square_{\dot{\alpha} \grave{\beta}}=\left(\gamma^{\alpha \beta}\right)_{\dot{\alpha} \grave{\beta}} \Delta_{\alpha \beta}=\left(\square_{\grave{\imath \imath}}, \square_{a \grave{a}}\right),
$$

with h- and v-components,

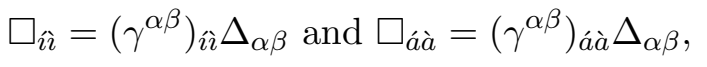

being symmetric or antisymmetric in dependence of corresponding values of dimensions $n$ and $m$. Considering the actions of operator (31) on $\mathrm{d}-$ spinors $\pi^{\hat{\gamma}}$ and $\mu_{\hat{\gamma}}$ we introduce the d-spinor curvature $X_{\tau \dot{\alpha} \dot{\beta}}^{\hat{\gamma}}$ satisfying the equations

$$
\square_{\dot{\alpha} \grave{\beta}} \pi^{\dot{\gamma}}=X_{\tau \dot{\tau} \dot{\beta}}^{\dot{\gamma}} \pi^{\hat{\tau}}
$$

and

$$
\square_{\alpha \grave{\beta}} \mu_{\dot{\gamma}}=X_{\dot{\gamma} \dot{\alpha} \dot{\beta}}^{\dot{\tau}} \mu_{\dot{\tau}} .
$$

The gravitational d-spinor $\Psi_{\tau \dot{\tau} \dot{\gamma} \dot{\alpha} \hat{\beta}}$ is defined by a corresponding symmetrization of d-spinor indices:

$$
\Psi_{\tau \dot{\gamma} \dot{\alpha} \dot{\beta}}=X_{(\tau \dot{\tau} \dot{\gamma} \dot{\alpha}) \grave{\beta}}
$$

We note that d-spinor tensors $X_{\dot{\gamma} \alpha \dot{\alpha}}^{\hat{\tau}^{\prime}}$ and $\Psi_{\dot{\tau} \dot{\gamma} \dot{\alpha} \dot{\beta}}$ are transformed into similar 2 -spinor objects if the $\mathrm{N}$-connection vanishes and the spinor constuctions are defined in global form on $\mathbf{V}$ [19, 20].

Putting $\mathbf{e}_{\dot{\gamma}}{ }^{\dot{\gamma}}$ instead of $\mu_{\dot{\gamma}}$ in (32) and using (33), we can express respectively the curvature and gravitational $\mathrm{d}-$-spinors as

$$
X_{\dot{\gamma} \dot{\delta} \dot{\alpha} \dot{\beta}}=\mathbf{e}_{\hat{\gamma} \dot{\tau}} \square_{\alpha \grave{\alpha}} \mathbf{e}_{\dot{\delta}}^{\tilde{\tau}}
$$

and

$$
\Psi_{\delta \dot{\gamma} \dot{\alpha} \dot{\beta}}=\mathbf{e}_{\hat{\delta}(\hat{\tau}} \square_{\hat{\alpha}|\grave{\beta}|} \mathbf{e}_{\hat{\gamma})}^{\hat{\tau}}
$$

where we omit symmetrization on $\grave{\beta}$.

The d-spinor torsion $T_{\alpha \dot{\gamma} \dot{\beta}}$ is defined by using the d-spinor commutator (31) and equations

$$
\square_{\alpha \grave{\alpha}} f=T_{\alpha \dot{\alpha} \grave{\gamma}}^{\dot{\gamma}} \nabla \dot{\gamma} \grave{\gamma} f .
$$




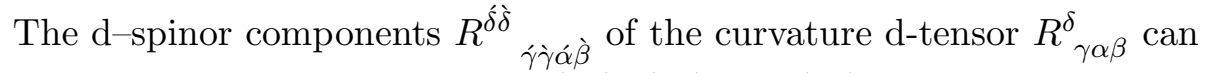
be computed by using the relations (30), (31) and (33) and the equations

$$
\left(\square_{\alpha \grave{\alpha}}-T_{\alpha \dot{\alpha} \grave{\gamma}}^{\dot{\gamma}} \nabla \dot{\gamma} \grave{\gamma}\right) V^{\delta \grave{\delta}}=R_{\hat{\gamma} \dot{\gamma} \dot{\alpha} \dot{\beta}}^{\hat{\delta} \grave{\gamma}} V^{\dot{\gamma} \dot{\gamma}},
$$

here d-vector $V^{\dot{\gamma} \hat{\gamma}}$ is considered as a product of d-spinors, i. e. $V^{\dot{\gamma} \grave{\gamma}}=\nu^{\hat{\gamma}} \mu^{\grave{\gamma}}$. We find

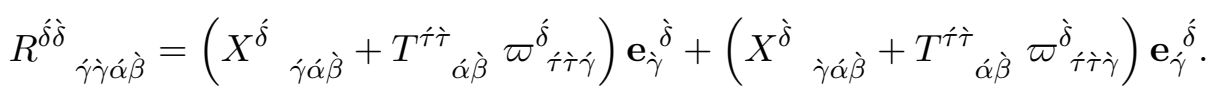

It is convenient to use this $\mathrm{d}$-spinor expression for the curvature $\mathrm{d}$-tensor in order to get the $\mathrm{d}$-spinor components of the Ricci $\mathrm{d}$-tensor,

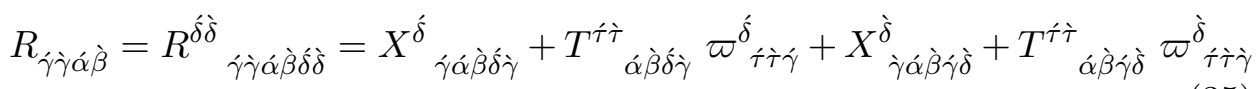

and this d-spinor decomposition of the scalar curvature $\overleftarrow{R}=R^{\alpha \dot{\alpha} \grave{\beta}}$,

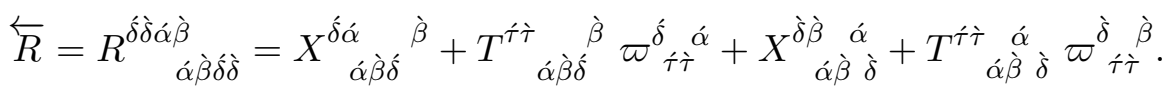

Finally, we write down the d-spinor components of the Einstein d-tensor $\mathbf{G}_{\gamma \beta}$,

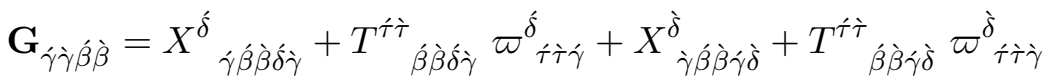

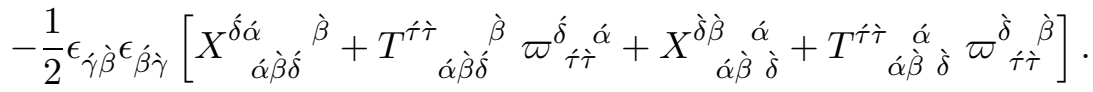

It should be noted that further reductions of (35) and (36) depend on dimensions $n$ and $m$ of the, respectively, $\mathrm{h}-$ and $\mathrm{v}-$ subspaces, and that the symmetry properties are defined by the $\epsilon$-objects. On Clifford $\mathrm{N}$-algebroids, such formulas have to be considered for anchored v-derivatives (15) and (17) (for d-spinor considerations, we have to apply spinor anchors (10) and (12)), for instance, in the case of canonical $\mathrm{d}$-connections and their spinor variants (29).

\section{$3 \quad$ Field Equations and Lie Algebroids}

Lie algebroid structures can be modelled as spacetime geometries with generalized symmetries (defined by anchors and Lie algebra commutators and nontrivial $\mathrm{N}$-connection structure) 22. It is possible to extend the constructions on Clifford $\mathrm{N}$-algebroids by introducing spinor variables. In this section we shall analyze the basic field equations for gravitational and matter field interactions modelled on $\mathrm{N}$-anholonomic manifolds and Clifford $\mathrm{N}$-algebroids. 


\subsection{The Dirac operator on $\mathrm{N}$-anholonomic spaces}

The aim of this section is to elucidate the possibility of definition of Dirac operators for general $\mathrm{N}$-anholonomic manifolds. It should be noted that such geometric constructions depend on the type of linear connections which are used for the complete definition of the Dirac operator. They are metric compatible and $\mathrm{N}$-adapted if the canonical $\mathrm{d}$-connection is used (we can similarly use any its deformation resulting in a metric compatible $\mathrm{d}-$ connection).

\subsubsection{Noholonomic vielbeins and spin $d-$ connections}

For a local dual coordinate basis $e^{\underline{i}} \doteq d x^{\underline{i}}$ on a manifold $M$, $\operatorname{dim} M=n$, we may respectively introduce certain classes of orthonormalized vielbeins and the $\mathrm{N}$-adapted vielbeins (depending both on the base coordinates $x \doteq x^{i}$ and some "fiber" coordinates $\left.y \doteq y^{a}\right)$

$$
e^{\hat{\imath}} \doteq e_{\underline{i}}^{\hat{\imath}}(x, y) e^{\underline{i}} \text { and } e^{i} \doteq e_{\underline{i}}^{i}(x, y) e^{\underline{i}}
$$

where

$$
g^{\underline{i}}(x, y) e_{\underline{i}}^{\hat{\imath}}(x, y) e_{\underline{j}}^{\hat{\jmath}}(x, y)=\delta^{\hat{\imath} \hat{\jmath}} \text { and } g^{\underline{i} \underline{j}}(x, y) e_{\underline{i}}^{i}(x, y) e_{\underline{j}}^{j}(x, y)=g^{i j}(x, y) .
$$

We define the the algebra of Dirac's gamma matrices (in brief, $\mathrm{h}$-gamma matrices defined by self-adjoints matrices $M_{k}(\mathbb{C})$ where $k=2^{n / 2}$ is the dimension of the irreducible representation of $\mathbb{C l}(M)$ for even dimensions, or of $\mathbb{C l}(M)^{+}$for odd dimensions) from the relation

$$
\gamma^{\hat{\imath}} \gamma^{\hat{\jmath}}+\gamma^{\hat{\jmath}} \gamma^{\hat{\imath}}=2 \delta^{\hat{\imath} \hat{\jmath}} \mathbb{I} \text {. }
$$

We can consider the action of $d x^{i} \in \mathbb{C l}(M)$ on a spinor $\psi \in S$ via representations

$$
{ }^{-} c\left(d x^{\hat{\imath}}\right) \doteq \gamma^{\hat{\imath}} \text { and }{ }^{-} c\left(d x^{i}\right) \psi \doteq \gamma^{i} \psi \equiv e^{i}{ }_{\hat{\imath}} \gamma^{\hat{\imath}} \psi .
$$

For any type of spaces $T_{x} M, T M$ or $\mathbf{V}$ possessing a local (in any point) or global fibered structure and enabled with a $\mathrm{N}$-connection structure, we can introduce similar definitions of the gamma matrices following algebraic relations and metric structures on fiber subspaces,

$$
e^{\hat{a}} \doteq e_{\underline{a}}^{\hat{a}}(x, y) e^{\underline{a}} \text { and } e^{a} \doteq e_{\underline{a}}^{a}(x, y) e^{\underline{a}},
$$

where

$$
g^{\underline{a b}}(x, y) e_{\underline{a}}^{\hat{a}}(x, y) e_{\underline{b}}^{\hat{b}}(x, y)=\delta^{\hat{a} \hat{b}} \text { and } g^{\underline{a b}}(x, y) e_{\underline{a}}^{a}(x, y) e_{\underline{b}}^{b}(x, y)=h^{a b}(x, y) .
$$

Similarly, we define the algebra of Dirac's matrices related to typical fibers (in brief, v-gamma matrices described by self-adjoints matrices $M_{k}^{\prime}(\mathbb{C})$ 
where $k^{\prime}=2^{m / 2}$ is the dimension of the irreducible representation of $\mathbb{C l}(F)$ for even dimensions, or of $\mathbb{C l}(F)^{+}$for odd dimensions, of the typical fiber $F$ ) from the relation

$$
\gamma^{\hat{a}} \gamma^{\hat{b}}+\gamma^{\hat{b}} \gamma^{\hat{a}}=2 \delta^{\hat{a} \hat{b}} \mathbb{I}
$$

The formulas (38) and (41) are respectively the $\mathrm{h}$ - and $\mathrm{v}$-components of the relation (18) (with redefined the coefficients which is more convenient for further constructions). The action of $d y^{a} \in \mathbb{C l}(F)$ on a spinor ${ }^{\star} \psi \in{ }^{\star} S$ is considered via representations

$$
{ }^{\star} c\left(d y^{\hat{a}}\right) \doteq \gamma^{\hat{a}} \text { and }{ }^{\star} c\left(d y^{a}\right){ }^{\star} \psi \doteq \gamma^{a}{ }^{\star} \psi \equiv e^{a}{ }_{\hat{a}} \gamma^{\hat{a}}{ }^{\star} \psi \text {. }
$$

We note that additionally to formulas (39) and (42) we may write respectively

$$
c\left(d x^{\underline{i}}\right) \psi \doteq \gamma^{\underline{i}} \psi \equiv e^{\underline{i}}{ }_{\hat{\imath}} \gamma^{\hat{\imath}} \psi \text { and } c\left(d y^{\underline{a}}\right)^{\star} \psi \doteq \gamma^{\underline{a}}{ }^{\star} \psi \equiv e^{\underline{a}}{ }_{\hat{a}} \gamma^{\hat{a} \star} \psi
$$

but such operators are not adapted to the $\mathrm{N}$-connection structure.

A more general gamma matrix calculus with distinguished gamma matrices (in brief, d-gamma matrices) can be elaborated for any $\mathrm{N}$-anholonomic manifold $\mathbf{V}$ provided with $\mathrm{d}$-metric structure $\mathbf{g}=\left[g,{ }^{\star} g\right]$ and for $\mathrm{d}$-spinors $\breve{\psi} \doteq\left(\psi,{ }^{\star} \psi\right) \in \mathbf{S} \doteq\left(S,{ }^{\star} S\right)$. Firstly, we should write in a unified form, related to a d-metric (11), the formulas (37) and (40),

$$
e^{\hat{\alpha}} \doteq e_{\underline{\alpha}}^{\hat{\alpha}}(u) e^{\underline{\alpha}} \text { and } e^{\alpha} \doteq e_{\underline{\alpha}}^{\alpha}(u) e^{\underline{\alpha}},
$$

where

$$
g^{\underline{\alpha} \underline{\beta}}(u) e_{\underline{\alpha}}^{\hat{\alpha}}(u) e_{\underline{\beta}}^{\hat{\beta}}(u)=\delta^{\hat{\alpha} \hat{\beta}} \text { and } g^{\underline{\alpha}} \underline{\beta}(u) e_{\underline{\alpha}}^{\alpha}(u) e_{\underline{\beta}}^{\beta}(u)=g^{\alpha \beta}(u) .
$$

The second step, is to consider gamma d-matrix relations (unifying (38) and (41) )

$$
\gamma^{\hat{\alpha}} \gamma^{\hat{\beta}}+\gamma^{\hat{\beta}} \gamma^{\hat{\alpha}}=2 \delta^{\hat{\alpha} \hat{\beta}} \mathbb{I},
$$

with the action of $d u^{\alpha} \in \mathbb{C} l(\mathbf{V})$ on a d-spinor $\breve{\psi} \in \mathbf{S}$ resulting in distinguished irreducible representations (unifying (39) and (42))

$$
\mathbf{c}\left(d u^{\hat{\alpha}}\right) \doteq \gamma^{\hat{\alpha}} \text { and } \mathbf{c}=\left(d u^{\alpha}\right) \breve{\psi} \doteq \gamma^{\alpha} \breve{\psi} \equiv e_{\hat{\alpha}}^{\alpha} \gamma^{\hat{\alpha}} \breve{\psi}
$$

which allows to write

$$
\gamma^{\alpha}(u) \gamma^{\beta}(u)+\gamma^{\beta}(u) \gamma^{\alpha}(u)=2 g^{\alpha \beta}(u) \mathbb{I} .
$$

In the canonical representation, we can write in irreducible form $\breve{\gamma} \doteq \gamma \oplus{ }^{\star} \gamma$ and $\breve{\psi} \doteq \psi \oplus{ }^{\star} \psi$, for instance, by using block type of $\mathrm{h}$ - and $\mathrm{v}$-matrices, or, writing alternatively as couples of gamma and/or $\mathrm{h}-$ and $\mathrm{v}$-spinor objects written in $\mathrm{N}$-adapted form,

$$
\gamma^{\alpha} \doteq\left(\gamma^{i}, \gamma^{a}\right) \text { and } \breve{\psi} \doteq\left(\psi,{ }^{\star} \psi\right)
$$


The decomposition (46) holds with respect to a N-adapted vielbein (3). We also note that for a spinor calculus, the indices of spinor objects should be treated as abstract spinorial ones possessing certain reducible, or irreducible, properties depending on the space dimension. For simplicity, we shall consider that spinors like $\breve{\psi}, \psi,{ }^{\star} \psi$ and all type of gamma objects can be enabled with corresponding spinor indices running certain values which are different from the usual coordinate space indices.

The spin connection $\nabla^{S}$ for the Riemannian manifolds is induced by the Levi-Civita connection ${ }^{\nabla} \Gamma$,

$$
\nabla^{S} \doteq d-\frac{1}{4} \nabla_{\Gamma^{i}{ }_{j k} \gamma_{i} \gamma^{j}} d x^{k}
$$

On $\mathrm{N}$-anholonomic spaces, it is possible to define spin connections which are $\mathrm{N}$-adapted by replacing the Levi-Civita connection by any $\mathrm{d}$-connection.

The canonical spin $\mathrm{d}$-connection is defined by the canonical d-connection,

$$
\widehat{\nabla}^{\mathbf{S}} \doteq \delta-\frac{1}{4} \widehat{\boldsymbol{\Gamma}}_{\beta \mu}^{\alpha} \gamma_{\alpha} \gamma^{\beta} \delta u^{\mu}
$$

where the absolute differential $\delta$ acts in $\mathrm{N}$-adapted form resulting in 1 -forms decomposed with respect to $\mathrm{N}$-elongated differentials $\delta u^{\mu}=\left(d x^{i}, \delta y^{a}\right)$ (2).

We note that the canonical spin $\mathrm{d}$-connection $\widehat{\nabla}^{\mathbf{S}}$ is metric compatible and contains nontrivial $\mathrm{d}$-torsion coefficients induced by the $\mathrm{N}$-anholonomy relations. It is possible to introduce more general spin $\mathrm{d}-$-connections $\mathbf{D}^{\mathbf{S}}$ by using the same formula (49) but for arbitrary metric compatible $\mathrm{d}$ connection $\Gamma_{\beta \mu}^{\alpha}$. For the spaces provided with generic off-diagonal metric structure (11) on a $\mathrm{N}$-anholonomic manifold, there is a canonical spin $\mathrm{d}-$-connection (49) induced by the off-diagonal metric coefficients with nontrivial $N_{i}^{a}$ and associated nonholonomic frames in gravity theories.

In a particular case of $\mathrm{N}$-anholonomic manifolds of even dimensions, we can define, for instance, the canonical spin $\mathrm{d}$-connections for a local modelling of a tangent bundle space with the canonical d-connection $\widehat{\boldsymbol{\Gamma}}^{\gamma}{ }_{\alpha \beta}=$ $\left(\widehat{L}_{j k}^{i}, \widehat{B}_{j k}^{i}\right)$. The N-connection structure $N_{i}^{j}$ states a global h-and v-splitting of the spin $\mathrm{d}$-connection operators, for instance,

$$
\widehat{\nabla} \doteq \delta-\frac{1}{4} \widehat{L}^{i}{ }_{j k} \gamma_{i} \gamma^{j} d x^{k}-\frac{1}{4} \widehat{B}_{b c}^{a} \gamma_{a} \gamma^{b} \delta y^{c} .
$$

So, any spin $\mathrm{d}$-connection is a d-operator with conventional splitting of action like $\nabla^{(\mathbf{S})} \equiv\left({ }^{-} \nabla^{(\mathbf{S})},{ }^{\star} \nabla^{(\mathbf{S})}\right)$, or $\nabla \equiv\left({ }^{-} \nabla,{ }^{\star} \nabla\right)$. For instance, for $\widehat{\nabla} \equiv\left({ }^{-} \widehat{\nabla},{ }^{\star} \widehat{\nabla}\right)$, the operators ${ }^{-} \widehat{\nabla}$ and ${ }^{\star} \widehat{\nabla}$ act respectively on a $\mathrm{h}-$ spinor $\psi$ as

$$
-\widehat{\nabla} \psi \doteq d x^{i} \frac{\delta \psi}{\partial x^{i}}-d x^{k} \frac{1}{4} \widehat{L}^{i}{ }_{j k} \gamma_{i} \gamma^{j} \psi
$$

and

$$
{ }^{\star} \widehat{\nabla} \psi \doteq \delta y^{a} \frac{\partial \psi}{\partial y^{a}}-\delta y^{c} \frac{1}{4} \widehat{B}_{b c}^{a} \gamma_{a} \gamma^{b} \psi
$$


being defined by the canonical d-connection, which (in its turn) is completely defined by $N_{i}^{j}(x, y)$ and $g_{i j}(x, y)$.

The operators (51) can be adapted to the Lie algebroid structure by anchoring the partial v-derivatives. For instance,

$$
\begin{aligned}
\frac{\delta \psi}{\partial x^{i}}\left(x^{k}, y^{b}\left(x^{j}\right)\right) & =\frac{\partial \psi}{\partial x^{i}}-N_{i}^{a} \frac{\partial \psi}{\partial y^{a}}=\left(\frac{\partial \psi}{\partial x^{i}}-N_{i}^{a} \rho_{a}^{k}\left(x^{j}\right) \frac{\partial \psi}{\partial x^{k}}\right) \\
& =\left(\frac{\partial \psi}{\partial x^{i}}-{ }^{\rho} N_{i}^{k} \frac{\partial \psi}{\partial x^{k}}\right)\left(x^{k}, y^{b}\left(x^{j}\right)\right)
\end{aligned}
$$

where the anchor $\rho_{a}^{k}$ (7) induce a N-connection ${ }^{\rho} N_{i}^{k} \doteqdot N_{i}^{a} \rho_{a}^{k}$. We can also perform a N-adapted Clifford algebroid calculus by using the "boldface" algebroid $\widehat{\rho}_{a}^{j}$ (15) with explicit dependence on variables $y^{b}$,

$$
\begin{aligned}
\frac{\delta \psi}{\partial x^{i}}\left(x^{k}, y^{b}\right) & =\frac{\partial \psi}{\partial x^{i}}-N_{i}^{a} e_{a} \psi=\left(\frac{\partial \psi}{\partial x^{i}}-N_{i}^{a} \widehat{\rho}_{a}^{k} \frac{\partial \psi}{\partial x^{k}}\right) \\
& =\left(\frac{\partial \psi}{\partial x^{i}}-{ }^{\rho} \hat{N}_{i}^{k} \frac{\partial \psi}{\partial x^{k}}\right)
\end{aligned}
$$

for ${ }^{\rho} \hat{N}_{i}^{k}=N_{i}^{a} \widehat{\rho}_{a}^{k}$. Such anchoring of partial/N-elongated derivatives has to be considered for the canonical d-connection $\widehat{L}^{i}{ }_{j k}$ and $\widehat{B}_{b c}^{a}$.

\subsubsection{Dirac $\mathrm{d}$-operators}

We consider a vector bundle $\mathbf{E}$ on an $\mathrm{N}$-anholonomic manifold $M$ (with two compatible $\mathrm{N}-$ connections defined as $\mathrm{h}-$ and $\mathrm{v}-$ splittings of $T \mathbf{E}$ and $T M)$ ). A d-connection

$$
\mathcal{D}: \operatorname{Sec}^{\infty}(\mathbf{E}) \rightarrow \operatorname{Sec}^{\infty}(\mathbf{E}) \otimes \Omega^{1}(M)
$$

preserves by parallelism splitting of the tangent total and base spaces and satisfy the Leibniz condition

$$
\mathcal{D}(f \sigma)=f(\mathcal{D} \sigma)+\delta f \otimes \sigma
$$

for any $f \in C^{\infty}(M)$, and $\sigma \in \operatorname{Sec}^{\infty}(\mathbf{E})$ and $\delta$ defining an $\mathrm{N}$-adapted exterior calculus by using N-elongated operators (3) and (2) which emphasize dforms instead of usual forms on $M$, with the coefficients taking values in E.

The metricity and Leibniz conditions for $\mathcal{D}$ are written respectively

$$
\mathbf{g}(\mathcal{D X}, \mathbf{Y})+\mathbf{g}(\mathbf{X}, \mathcal{D} \mathbf{Y})=\delta[\mathbf{g}(\mathbf{X}, \mathbf{Y})]
$$

for any $\mathbf{X}, \mathbf{Y} \in \chi(M)$, and

$$
\mathcal{D}(\sigma \beta) \doteq \mathcal{D}(\sigma) \beta+\sigma \mathcal{D}(\beta),
$$


for any $\sigma, \beta \in \operatorname{Sec}^{\infty}(\mathbf{E})$.

For local computations, we may define the corresponding coefficients of the geometric $\mathrm{d}$-objects and write

$$
\mathcal{D} \sigma_{\breve{\beta}} \doteq \boldsymbol{\Gamma}_{\check{\beta} \mu}^{\check{\alpha}} \sigma_{\check{\alpha}} \otimes \delta u^{\mu}=\boldsymbol{\Gamma}_{\check{\beta} i}^{\check{\alpha}} \sigma_{\check{\alpha}} \otimes d x^{i}+\boldsymbol{\Gamma}_{\check{\beta} a}^{\check{\alpha}} \sigma_{\check{\alpha}} \otimes \delta y^{a},
$$

where fiber "inverse hat" indices, in their turn, may split $\check{\alpha} \doteq(\check{\imath}, \check{a})$ if any Nconnection structure is defined on $T \mathbf{E}$. For some constructions of particular interest, we can take $\mathbf{E}=T^{*} \mathbf{V},=T^{*} V_{(g)}$ and/or any Clifford d-algebra $\mathbf{E}=\mathbb{C l}(\mathbf{V}), \mathbb{C l}\left(V_{(g)}\right), \ldots$ with a corresponding treating of "acute" indices to of $\mathrm{d}$-tensor and/or d-spinor type as well when the d-operator $\mathcal{D}$ transforms into respective d-connection $\mathbf{D}$ and spin d-connections $\widehat{\nabla}^{\mathbf{S}}$ (49), $\widehat{\nabla}^{(g)} \ldots$. All such, adapted to the $\mathrm{N}$-connections, computations are similar for both $\mathrm{N}$-anholonomic (co) vector and spinor bundles.

The respective actions of the Clifford d-algebra and the Clifford-Lagrange algebra can be transformed into maps $\operatorname{Sec}^{\infty}(\mathbf{S}) \otimes \operatorname{Sec}(\mathbb{C l}(\mathbf{V}))$ and $\operatorname{Sec}^{\infty}\left(S_{(g)}\right) \otimes \operatorname{Sec}\left(\mathbb{C l}\left(V_{(g)}\right)\right)$ to $\operatorname{Sec}^{\infty}(\mathbf{S})$ and, respectively, $\operatorname{Sec}^{\infty}\left(S_{(g)}\right)$ by considering maps of type (39) and (45)

$$
\widehat{\mathbf{c}}(\breve{\psi} \otimes \mathbf{a}) \doteq \mathbf{c}(\mathbf{a}) \breve{\psi} \text { and } \widehat{c}(\psi \otimes a) \doteq c(a) \psi .
$$

Definition 3.1 The Dirac d-operator (or Dirac $N$-anholonomic operator) on a spin $N$-anholonomic manifold $(\mathbf{V}, \mathbf{S}, J)$ (or on a spin manifold $\left.\left(M_{(g)}, S_{(g)}, J\right)\right)$ is defined

$$
\begin{aligned}
\mathbb{D} & \doteq-i\left(\widehat{\mathbf{c}} \circ \nabla^{\mathbf{S}}\right) \\
& =\left({ }^{-} \mathbf{D}=-i\left({ }^{-\widehat{c} \circ}{ }^{-} \nabla^{\mathbf{S}}\right),{ }^{\star} \mathbb{D}=-i\left({ }^{\star} \widehat{c} \circ{ }^{\star} \nabla^{\mathbf{S}}\right)\right) \\
\left({ }_{(g)} \mathbb{D}\right. & \left.\doteq-i\left(\widehat{c} \circ \nabla^{(g)}\right)\right) \\
& =\left({ }_{(g)}{ }^{-} \mathbf{D}=-i\left({ }^{-} \widehat{c} \circ{ }^{-} \nabla^{(g)}\right),(g)\right.
\end{aligned}
$$

Such $N$-adapted Dirac d-operators are called canonical and denoted $\widehat{\mathbf{D}}=$ $\left(-\widehat{\mathbb{D}},{ }^{\star} \widehat{\mathbf{D}}\right)\left({ }_{(g)} \widehat{\mathbf{D}}=\left({ }_{(g)}{ }^{-} \widehat{\mathbf{D}},(g)^{\star} \widehat{\mathbb{D}}\right)\right)$ if they are defined for the canonical $d-$ connection and respective spin $d-$-connection (49).

Now we can formulate the

Theorem 3.1 Let $(\mathbf{V}, \mathbf{S}, J)\left(\left(M_{(g)}, S_{(g)}, J\right)\right.$ be a spin $N$-anholonomic manifold. There is the canonical Dirac d-operator (Dirac $N$-anholonomic operator) defined by the almost Hermitian spin d-operator

$$
\widehat{\nabla}^{\mathbf{S}}: \operatorname{Sec}^{\infty}(\mathbf{S}) \rightarrow \operatorname{Sec}^{\infty}(\mathbf{S}) \otimes \Omega^{1}(\mathbf{V})
$$

(N-anholonomic spin operator

$$
\left.\widehat{\nabla}^{(g)}: \operatorname{Sec}^{\infty}\left(S_{(g)}\right) \rightarrow \operatorname{Sec}^{\infty}\left(S_{(g)}\right) \otimes \Omega^{1}\left(M_{(g)}\right)\right)
$$


commuting with $J$ and satisfying the conditions

$$
\left(\widehat{\nabla}^{\mathbf{S}} \breve{\psi} \mid \breve{\phi}\right)+\left(\breve{\psi} \mid \widehat{\nabla}^{\mathbf{S}} \breve{\phi}\right)=\delta(\breve{\psi} \mid \breve{\phi})
$$

and

$$
\widehat{\nabla}^{\mathbf{S}}(\mathbf{c}(\mathbf{a}) \breve{\psi})=\mathbf{c}(\widehat{\mathbf{D}} \mathbf{a}) \breve{\psi}+\mathbf{c}(\mathbf{a}) \widehat{\nabla}^{\mathbf{S}} \breve{\psi}
$$

for $\mathbf{a} \in \mathbb{C l}(\mathbf{V})$ and $\breve{\psi} \in \operatorname{Sec}^{\infty}(\mathbf{S})$

$$
\left(\left(\widehat{\nabla}^{(g)} \breve{\psi} \mid \breve{\phi}\right)+\left(\breve{\psi} \mid \widehat{\nabla}^{(g)} \breve{\phi}\right)=\delta(\breve{\psi} \mid \breve{\phi})\right.
$$

and

$$
\widehat{\nabla}^{(g)}(\mathbf{c}(\mathbf{a}) \breve{\psi})=\mathbf{c}(\widehat{\mathbf{D}} \mathbf{a}) \breve{\psi}+\mathbf{c}(\mathbf{a}) \widehat{\nabla}^{(g)} \breve{\psi}
$$

for $\mathbf{a} \in \mathbb{C l}\left(M_{(g)}\right)$ and $\breve{\psi} \in \operatorname{Sec}^{\infty}\left(S_{(g)}\right)$ determined by the metricity (52) and Leibnitz (53) conditions.

Proof. We sketch the main idea of such a proof being similar to that given in Ref. [24, Theorem 9.8, for the Levi-Civita connection, see also Ref. [25]. In our case, we have to extend the constructions for $\mathrm{d}$-metrics and canonical $\mathrm{d}$-connections by applying $\mathrm{N}$-elongated operators for differentials and partial derivatives and distinguishing the formulas into $\mathrm{h}-$ and $\mathrm{v}$-irreducible components.

The canonical Dirac d-operator has very similar properties for spin $\mathrm{N}$ anholonomic manifolds and spin Lagrange, or Finsler spaces [11, 12, 13, 7].

\subsection{Field equations on $\mathrm{N}$-anholonomic manifolds}

The general idea is to formulate such equations with respect to a nonholonomic frame on (pesudo) Riemann-Cartan space. Then the constructions are $\mathrm{N}$-adapted by considering $\mathrm{N}$-elongated frames. For Lie/Clifford $\mathrm{N}-$ algebroid structures, we have to anchor the formulas.

\subsubsection{Scalar field on $\mathrm{N}$-anholonomic manifolds}

Let $\varphi(u)=\left(\varphi_{1}(u), \varphi_{2}(u) ; \ldots, \varphi_{k}(u)\right)$ be a complex k-component scalar field of mass $\mu$ on a $\mathrm{N}$-anholonomic manifold $\mathbf{V}$. The d-covariant generalization of the conformally invariant (in the massless case) scalar field equation [19, 20. can be defined by using the d'Alambert operator $\square=\mathbf{D}^{\alpha} \mathbf{D}_{\alpha}$, where $\mathbf{D}_{\alpha}$ is a metric compatible d-connection,

$$
\left(\square+\frac{n+m-2}{4(n+m-1)} \overleftarrow{R}+\mu^{2}\right) \varphi(u)=0
$$

We have to elongate the covariant d-operator, $\breve{D}_{\alpha}=\mathbf{D}_{\alpha}+i e A_{\alpha}$, and take into account the d-vector current

$$
J_{\alpha}^{(0)}(u)=i\left(\left(\bar{\varphi}(u) \mathbf{D}_{\alpha} \varphi(u)-\mathbf{D}_{\alpha} \bar{\varphi}(u)\right) \varphi(u)\right)
$$


if there are considered interactions with the electromagnetic field ( $\mathrm{d}$-vector potential $A_{\alpha}$ ), where $e$ is the electromagnetic constant, and a charged scalar field $\varphi$. The equations (58) are just the Euler equations for the Lagrangian

$$
\mathcal{L}^{(0)}(u)=\sqrt{|g|}\left[\mathbf{g}^{\alpha \beta} e_{\alpha} \bar{\varphi}(u) e_{\beta} \varphi(u)-\left(\mu^{2}+\frac{n+m-2}{4(n+m-1)}\right) \bar{\varphi}(u) \varphi(u)\right],
$$

where $|g|=\operatorname{det}\left|\mathbf{g}_{\alpha \beta}\right|$ and $\mathbf{e}_{\alpha}$ is defined by (3), and must be anchored for Lie algebroid structures.

The $\mathrm{N}$-adapted variations of the action with Lagrangian (59) on variables $\varphi(u)$ and $\bar{\varphi}(u)$ lead to the energy-momentum d-tensor,

$$
E_{\alpha \beta}^{(0, c)}(u)=\mathbf{e}_{\alpha} \bar{\varphi}(u) \mathbf{e}_{\beta} \varphi(u)+\mathbf{e}_{\beta} \bar{\varphi}(u) \mathbf{e}_{\alpha} \varphi(u)-\frac{1}{\sqrt{|g|}} \mathbf{g}_{\alpha \beta} \mathcal{L}^{(0)}(u),
$$

and a similar variation on the components of a d-metric (11) leads to a symmetric energy-momentum d-tensor,

$$
E_{\alpha \beta}^{(0)}(u)=E_{(\alpha \beta)}^{(0, c)}(u)-\frac{n+m-2}{2(n+m-1)}\left[\mathbf{R}_{(\alpha \beta)}+\mathbf{D}_{(\alpha} \mathbf{D}_{\beta)}-\mathbf{g}_{\alpha \beta} \square\right] \bar{\varphi}(u) \varphi(u) .
$$

We also conclude that the $\mathrm{N}$-connection results in a nonequivalence of energy-momentum d-tensors (60) and (61), nonsymmetry of the Ricci tensor, non-vanishing of the d-covariant derivation of the Einstein d-tensor,

$\mathbf{D}_{\alpha} \overleftarrow{\mathbf{G}}^{\alpha \beta} \neq 0$ and, in consequence, a corresponding modification of conservation laws on $\mathrm{N}$-anholonomic manifolds.

\subsubsection{Proca equations}

Let consider a d-vector field $\varphi_{\alpha}(u)$ with mass $\mu^{2}$ (Proca field) interacting with exterior gravitational field. From the Lagrangian

$$
\mathcal{L}^{(1)}(u)=\sqrt{|\mathbf{g}|}\left[-\frac{1}{2} \bar{f}_{\alpha \beta}(u) f^{\alpha \beta}(u)+\mu^{2} \bar{\varphi}_{\alpha}(u) \varphi^{\alpha}(u)\right]
$$

where

$$
f_{\alpha \beta}=\mathbf{D}_{\alpha} \varphi_{\beta}-\mathbf{D}_{\beta} \varphi_{\alpha},
$$

one follows the Proca equations on $\mathrm{N}$-anholonomic manifolds

$$
\mathbf{D}_{\alpha} f^{\alpha \beta}(u)+\mu^{2} \varphi^{\beta}(u)=0 .
$$

Equations (63) transform into a first type constraints for $\beta=0$. Acting with $\mathbf{D}_{\alpha}$ on (63), for $\mu \neq 0$ we obtain second type constraints

$$
\mathbf{D}_{\alpha} \varphi^{\alpha}(u)=0 \text {. }
$$


Putting (64) into (63) we obtain second order field equations with respect to $\varphi_{\alpha}$ :

$$
\square \varphi_{\alpha}(u)+\mathbf{R}_{\alpha \beta} \varphi^{\beta}(u)+\mu^{2} \varphi_{\alpha}(u)=0 .
$$

Anchoring of derivatives has to be considered for the operators $\mathbf{D}_{\alpha}$ and (as a consequence) for $\square$ and $\mathbf{R}_{\alpha \beta}$. The energy-momentum d-tensor and d-vector current following from the (65) can be written

$$
E_{\alpha \beta}^{(1)}(u)=-\mathbf{g}^{\varepsilon \tau}\left(\bar{f}_{\beta \tau} f_{\alpha \varepsilon}+\bar{f}_{\alpha \varepsilon} f_{\beta \tau}\right)+\mu^{2}\left(\bar{\varphi}_{\alpha} \varphi_{\beta}+\bar{\varphi}_{\beta} \varphi_{\alpha}\right)-\frac{\mathbf{g}_{\alpha \beta}}{\sqrt{|\mathbf{g}|}} \mathcal{L}^{(1)}(u) .
$$

and

$$
J_{\alpha}^{(1)}(u)=i\left(\bar{f}_{\alpha \beta}(u) \varphi^{\beta}(u)-\bar{\varphi}^{\beta}(u) f_{\alpha \beta}(u)\right) .
$$

For $\mu=0$ the d-tensor $f_{\alpha \beta}$ and the Lagrangian (62) are invariant with respect to gauge transforms of type

$$
\varphi_{\alpha}(u) \rightarrow \varphi_{\alpha}(u)+\delta_{\alpha} \Lambda(u),
$$

where $\Lambda(u)$ is a d-differentiable scalar function, and we obtain a variant of Maxwell theory on $\mathrm{N}$-anholonomic manifolds.

\subsubsection{Gravitons $\mathrm{N}$-anholonomic backgrounds}

Let un consider a massless d-tensor field $\mathbf{q}_{\alpha \beta}(u)$ as a small perturbation of the d-metric $\mathbf{g}_{\alpha \beta}(u)$. Considering, for simplicity, a torsionless background we have the Fierz-Pauli equations

$$
\square \mathbf{q}_{\alpha \beta}(u)+2 \mathbf{R}_{\tau \alpha \beta \nu}(u) \mathbf{q}^{\tau \nu}(u)=0
$$

and d-gauge conditions

$$
D_{\alpha} \mathbf{q}_{\beta}^{\alpha}(u)=0, \quad \mathbf{q}(u) \equiv \mathbf{q}_{\beta}^{\alpha}(u)=0,
$$

where $\mathbf{R}_{\tau \alpha \beta \nu}(u)$ is curvature d-tensor (these formulae can be obtained by using a perturbation formalism with respect to $\mathbf{q}_{\alpha \beta}(u)$; in our case we must take into account the distinguishing of geometrical objects.

We note that we can rewrite d-tensor formulas (58)-(67) into similar d-spinor ones by considering spinor variables.

\subsubsection{N-anholonomic Dirac equation}

Let denote the Dirac d-spinor field by $\psi(u)=\left(\psi^{\dot{\alpha}}(u)\right)$ and consider as the generalized Lorentz transforms the group of automorphysm of the metric $g_{\widehat{\alpha} \widehat{\beta}}$ (for a $\mathrm{N}$-adapted frame decomposition of d-metric). The d-covariant derivation of field $\psi(u)$ is written as

$$
\vec{\nabla}_{\alpha} \psi=\left[\mathbf{e}_{\alpha}+\frac{1}{4} C_{\widehat{\alpha} \widehat{\beta} \widehat{\gamma}}(u) l_{\alpha}^{\widehat{\alpha}}(u) \gamma^{\widehat{\beta}} \gamma^{\widehat{\gamma}}\right] \psi,
$$


where coefficients $C_{\widehat{\alpha} \widehat{\beta} \widehat{\gamma}}=\left(\mathbf{D}_{\gamma} l_{\widehat{\alpha}}^{\alpha}\right) l_{\widehat{\beta} \alpha} l_{\widehat{\gamma}}^{\gamma}$ generalize for N-anholonomic spaces the corresponding Ricci coefficients on Riemannian spaces. Using $\gamma$-objects $\gamma^{\alpha}(u)$ (see (19)), we define the Dirac equations on N-anholonomic manifolds:

$$
\left(i \gamma^{\alpha}(u) \vec{\nabla}_{\alpha}-\mu\right) \psi=0
$$

which are the Euler equations for the Lagrangian

$$
\begin{aligned}
\mathcal{L}^{(1 / 2)}(u)= & \sqrt{|g|}\left\{\left[\psi^{+}(u) \gamma^{\alpha}(u) \vec{\nabla}_{\alpha} \psi(u)\right.\right. \\
& \left.\left.-\left(\vec{\nabla}_{\alpha} \psi^{+}(u)\right) \gamma^{\alpha}(u) \psi(u)\right]-\mu \psi^{+}(u) \psi(u)\right\},
\end{aligned}
$$

where $\psi^{+}(u)$ is the complex conjugation and transposition of the column $\psi(u)$. We have to consider anchoring of the operator $\vec{\nabla}_{\alpha}$ on the N-anholonomic manifolds.

From (70), we obtain the d-metric energy-momentum d-tensor

$$
\begin{aligned}
E_{\alpha \beta}^{(1 / 2)}(u)= & \frac{i}{4}\left[\psi^{+}(u) \gamma_{\alpha}(u) \vec{\nabla}_{\beta} \psi(u)+\psi^{+}(u) \gamma_{\beta}(u) \vec{\nabla}_{\alpha} \psi(u)\right. \\
& \left.-\left(\vec{\nabla}_{\alpha} \psi^{+}(u)\right) \gamma_{\beta}(u) \psi(u)-\left(\vec{\nabla}_{\beta} \psi^{+}(u)\right) \gamma_{\alpha}(u) \psi(u)\right]
\end{aligned}
$$

and the d-vector source

$$
J_{\alpha}^{(1 / 2)}(u)=\psi^{+}(u) \gamma_{\alpha}(u) \psi(u) .
$$

We emphasize that interactions with exterior gauge fields can be introduced by changing the locally anisotropic partial derivation from (68) in this manner:

$$
e_{\alpha} \rightarrow e_{\alpha}+i e^{\star} B_{\alpha}
$$

where $e^{\star}$ and $B_{\alpha}$ are respectively the constant and the d-vector potential of gauge fields.

\subsubsection{Yang-Mills equations in $\mathrm{d}$-spinor form}

We consider a vector bundle $\mathcal{B}_{E}, \pi_{B}: \mathcal{B} \rightarrow \mathbf{V}$ on $\mathbf{V}$. Additionally to the $\mathrm{d}$-tensor and $\mathrm{d}$-spinor indices, we use capital Greek letters, $\Phi, \Upsilon, \Xi, \Psi, \ldots$ for fibre (of this bundle) indices (see details in [19, 20]). Let $\nabla_{\alpha}$ be, for simplicity, a torsionless, linear connection in $\mathcal{B}_{E}$ satisfying conditions:

$$
\begin{aligned}
\underline{\nabla}_{\alpha} & : \Upsilon^{\Theta} \rightarrow \Upsilon_{\alpha}^{\Theta} \quad\left[\text { or } \Xi^{\Theta} \rightarrow \Xi_{\alpha}^{\Theta}\right], \\
\underline{\nabla}_{\alpha}\left(\lambda^{\Theta}+\nu^{\Theta}\right) & =\underline{\nabla}_{\alpha} \lambda^{\Theta}+\underline{\nabla}_{\alpha} \nu^{\Theta}, \\
\underline{\nabla}_{\alpha}\left(f \lambda^{\Theta}\right) & =\lambda^{\Theta} \underline{\nabla}_{\alpha} f+f \underline{\nabla}_{\alpha} \lambda^{\Theta}, \quad f \in \Upsilon^{\Theta}\left[\text { or } \Xi^{\Theta}\right],
\end{aligned}
$$

where by $\Upsilon^{\Theta}\left(\Xi^{\Theta}\right)$ we denote the module of sections of the real (complex) v-bundle $\mathcal{B}_{E}$ provided with the abstract index $\Theta$. The curvature of connection $\underline{\nabla}_{\alpha}$ is defined as

$$
K_{\alpha \beta \Omega}{ }^{\Theta} \lambda^{\Omega}=\left(\underline{\nabla}_{\alpha} \underline{\nabla}_{\beta}-\underline{\nabla}_{\beta} \underline{\nabla}_{\alpha}\right) \lambda^{\Theta} .
$$


For Yang-Mills fields, as a rule, one considers that $\mathcal{B}_{E}$ is enabled with a unitary (complex) structure (complex conjugation changes mutually the upper and lower Greek indices). It is useful to introduce instead of $K_{\alpha \beta \Omega} \Theta$ a Hermitian matrix $F_{\alpha \beta \Omega}{ }^{\Theta}=i K_{\alpha \beta \Omega}{ }^{\Theta}$ connected with components of the Yang-Mills d-vector potential $B_{\alpha \Xi}{ }^{\Phi}$ according the formula:

$$
\frac{1}{2} F_{\alpha \beta \Xi} \Phi=\underline{\nabla}_{[\alpha} B_{\beta] \Xi}^{\Phi}-i B_{[\alpha|\Lambda|}^{\Phi} B_{\beta] \Xi}^{\Lambda},
$$

where the spacetime indices commute with capital Greek indices. The gauge transforms are written in the form:

$$
\begin{aligned}
B_{\alpha \Theta}{ }^{\Phi} & \mapsto B_{\alpha \widehat{\Theta}} \widehat{\widehat{\Phi}}=B_{\alpha \Theta}{ }^{\Phi} s_{\Phi}{ }^{\widehat{\Phi}} q_{\widehat{\Theta}}{ }^{\Theta}+i s_{\Theta}{ }^{\widehat{\Phi}} \underline{\nabla}_{\alpha} q_{\widehat{\Theta}}{ }^{\Theta}, \\
F_{\alpha \beta \Xi}{ }^{\Phi} & \mapsto F_{\alpha \beta \widehat{\Xi}}{ }^{\widehat{\Phi}}=F_{\alpha \beta \Xi}{ }^{\Phi} s_{\Phi} \widehat{\Phi}_{q_{\widehat{\Xi}}} \Xi
\end{aligned}
$$

where matrices $s_{\Phi} \widehat{\Phi}$ and $q_{\widehat{\Xi}} \Xi$ are mutually inverse (Hermitian conjugated in the unitary case). The Yang-Mills d-equations are written

$$
\begin{aligned}
& \underline{\nabla}^{\alpha} F_{\alpha \beta \Theta}^{\Psi}=J_{\beta \Theta}{ }^{\Psi}, \\
& \underline{\nabla}_{[\alpha} F_{\beta \gamma] \Theta} \Xi=0 .
\end{aligned}
$$

We must introduce deformations of connection of type, $\nabla_{\alpha}^{\star} \longrightarrow \underline{\nabla}_{\alpha}+P_{\alpha}$, (the deformation d-tensor $P_{\alpha}$ is induced by the torsion in the vector bundle $\left.\mathcal{B}_{E}\right)$ into the definition of the curvature of gauge fields (72) and motion equations (73) and (74) if the interactions are considered for nontrivial torsions.

\section{Conclusions and Outlook}

In this work we formulated a spinor approach to the geometry of nonholonomic spacetimes and classical field interactions with constraints possessing Lie algebroid symmetry. Such geometric constructions are performed for a special case of nonholonomic distributions defining nonlinear connection ( $\mathrm{N}$-connection) structures resulting in preferred classes of vielbein (frame) systems of reference. The main goals we have achieved are the following:

1. We gave an intrinsic formulation of the geometry of Clifford N-anholonomic structures. In addition, we investigated the $\mathrm{N}$-anholonomic spin structures (i. e. spinor nonholonomic spaces with associated Nconnection).

2. We defined and analyzed the main properties of the Dirac operator on $\mathrm{N}$-anholonomic manifolds. We showed how the formulas may be "anchored" in order to be considered on spacetimes with Lie/ Clifford algebroid symmetries. 
3. We formulated a geometric approach to field equations on N-anholonomic manifolds. There were considered the examples of scalar, Proca, graviton, spinor and gauge filed interactions when the formulas have a straightforward re-definition on Lie/Clifford $\mathrm{N}$-algebroids (i. e. spacetimes with algebroid symmetries and nonholonomic distributions).

Among the subjects we will study in forthcoming papers, we note the points:

- To construct exact solutions of the gravitational field equations in string gravity with nontrivial limits to general relativity, parametrized by generic off-diagonal metrics and nonholonomic frames and possessing Lie algebroid symmetries (the first examples of "gravitational" algebroids were analyzed in Ref. [2]).

- Certain extension of the metrics to configurations defining solutions of the Einstein-Dirac equations will be considered. We shall analyze the symmetries of such spacetimes and possible physical applications in modern gravity.

- In explicit form, we shall construct nonholonomically deformed metrics, with algebroid symmetries, describing locally anisotropic cosmological models, black holes, anholonomic wormholes, solitons and gravitational monopoles and instantons.

- To make a detailed investigation of classical field theories and their quantum deformations possessing nontrivial noncommutative symmetries and possible Lie/ Clifford algebroid structure.

Finally, we note that the method of anholonomic frames with associated $\mathrm{N}$-connection structure elaborated in Finsler geometry and further, in our works, applied to constructing exact solutions in gravity was applied in this paper for a study of Dirac operators on nonholonomic manifolds possessing Lie algebroid symmetry. The constructions can be extended for spacetimes with uncompactified extra dimensions and such investigations are regarded as interesting researches in modern physics and noncommutative geometry.

\section{Acknowledgments}

The author is grateful to the referee for hard work and constructive critics.

\section{References}

[1] S. Vacaru, Exact Solutions with Noncommutative Symmetries in Einstein and Gauge Gravity, J. Math. Phys. 46 (2005) 042503. 
[2] S. Vacaru, Einstein-Cartan Algebroids and Black Holes in Solitonic Backgrounds, gr-qc/ 0501057.

[3] F. Etayo, R. Santamaría and S. Vacaru, J. Math. Phys. 46, 032901 (2005) (17 pages).

[4] A. Cannas da Silva and A. Weinstein, Geometric Models for Noncommutative Algebras (Amer. Math. Soc., Providence, RI, 1999).

[5] R. Miron and M. Anastasiei, The Geometry of Lagrange Spaces: Theory and Applications (Kluwer, 1994).

[6] S. Vacaru, E. Gaburov and D. Gontsa, A Method of Constructing Off-Diagonal Solutions in Metric-Affine and String Gravity, hep-th/0310133 Chapter 2 in: Clifford and Riemann- Finsler Structures in Geometric Mechanics and Gravity, Selected Works, by S. Vacaru, P. Stavrinos, E. Gaburov and D. Gonţa. Differential Geometry - Dinamical Systems, Monograph 7 (Geometry Balkan Press, 2006); www.mathem.pub.ro/dgds/mono/va-t.pdf and gr-qc/0508023

[7] S. Vacaru, Nonholonomic Clifford Structures and Noncommutative Riemann-Finsler Geometry, math.DG/0408121, Chapter 15 in: Clifford and Riemann- Finsler Structures in Geometric Mechanics and Gravity, Selected Works, by S. Vacaru, P. Stavrinos, E. Gaburov and D. Gonţa. Differential Geometry - Dinamical Systems, Monograph 7 (Geometry Balkan Press, 2006); www.mathem.pub.ro/dgds/mono/va-t.pdf and gr-qc/0508023

[8] S. Vacaru and O. Tintareanu-Mircea, Anholonomic Frames, Generalized Killing Equations, and Anisotropic Taub NUT Spinning Spaces, Nucl. Phys. B 626 (2002) 239-264.

[9] S. Vacaru and F. C. Popa, Dirac Spinor Waves and Solitons in Anisotropic Taub-NUT Spaces, Class. Quant. Gravity, 18 (2001) 49214938 .

[10] S. Vacaru and N. Vicol, Nonlinear Connections and Spinor Geometry, Int. J. Math. and Math. Sciences 23 (2004) 1189-1237.

[11] S. Vacaru, Spinor Structures and Nonlinear Connections in Vector Bundles, Generalized Lagrange and Finsler Spaces, J. Math. Phys. 37 (1996) 508-523.

[12] S. Vacaru, Spinors and Field Interactions in Higher Order Anisotropic Spaces, JHEP 9809 (1998) 011.

[13] S. Vacaru and P. Stavrinos, Spinors and Space-Time Anisotropy (Athens University Press, Athens, Greece, 2002), gr-qc/0112028. 
[14] A. Weinstein, Lagrangian Mechanics and Groupoids. Fields Institute Communications, 7 (1996) 201-231.

[15] P. Libermann, Lie Algebroids and Mechanics, Archh. Math. (Brno) 32 (1996) 147-162.

[16] E. Martinez, Lagrangian Mechanics on Lie Algebroids, Acat Appl. Math. 67 (2001) 295-320.

[17] M. de Leon, J. C. Marrero and E. Martinez, Lagrangian Submanifolds and Dynamics on Lie Algebroids, math.DG/0407528.

[18] S. Vacaru, Gauge and Einstein Gravity from Non-Abelian Gauge Models on Noncommutative Spaces, Phys. Lett. B 498 (2001) 74.

[19] R. Penrose and W. Rindler, Spinors and Space-Time, vol. 1, Two-Spinor Calculus and Relativistic Fields (Cambridge University Press, Cambridge, 1984).

[20] R. Penrose and W. Rindler, Spinors and Space-Time, vol. 2, Spinor and Twistor Methods in Space-Time Geometry (Cambridge University Press, Cambridge, 1986).

[21] C. P. Luehr and M. Rosenbaum, Spinor Connections in General Relativity, J. Math. Phys. 15 (1974) 1120-1137.

[22] A. Salam and J. Strathdee, On Kaluza-Klein theory, Ann. Phys. (NY) 141 (1982) 316-352.

[23] J. M. Overduin and P. S. Wesson, Remarks on the Spinor Gauge Field Theory, Phys. Rep. 283 (1997) 303-378.

[24] J. M. Gracia-Bondia, J. C. Varilli and H. Figueroa, Elements of Noncommutative Geometry (Birkhauser, 2001).

[25] H. Schroeder, On the Definition of Geometric Dirac Operators, math. DG/ 0005239 .

[26] A. Ashtekar, New Hamiltonian Formulation of General Relativity, Phys. Rev. D36 (1987) 1587-1602.

[27] J. Kern, Lagrange Geometry, Arch. Math. 25 (1974) 438. 